\title{
Organocatalytic Synthesis of $\alpha$-Triphenylmethylamines from Diarylketimines and Phenols
}

\author{
Danijel Glavač, ${ }^{\ddagger}$ Nikola Topolovčan, ${ }^{\ddagger}$ Matija Gredičak* \\ Laboratory for Biomimetic Chemistry, Division of Organic Chemistry and Biochemistry, \\ Ruđer Bošković Institute, Bijenička cesta 54, 10000 Zagreb, Croatia.
}

\section{Abstract}
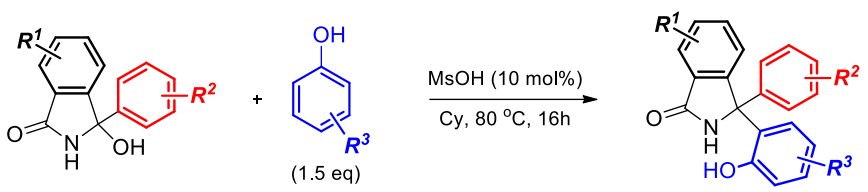

27 examples $18-96 \%$ yield

- wide substrate scope • metal-free conditions • easily accessible starting materials

Formal Betti reaction between variously substituted phenols and benzophenone-derived imines to afford $\alpha$-triphenylmethylamines is reported. Key to success of this transformation is the in situ generation of the reactive benzophenone iminium species under organocatalytic conditions. Different phenols reacted smoothly enabling the synthesis of an array of $\alpha$-triphenylmethylamines, which are highly valued structural motifs in bioactive molecules and chemical sensors. 
$\alpha$-Tertiary amines - a class of amines adjacent to a tertiary carbon atom - are an attractive structural subunit in bioactive molecules, ${ }^{1}$ chemical sensors ${ }^{2}$ and molecules used for subcellular imaging ${ }^{3}$ (Scheme 1). The usual strategy for their preparation is addition of carbon nucleophiles to ketimines. It follows that the most efficient approach for the synthesis of $\alpha$-triphenylmethylamines would be the direct C-arylation of benzophenone imines, as their parent ketones are either commercially available, or can be prepared by well-known synthetic routes. Although the arylation of alkyl-aryl imines is well developed and can provide access to $\alpha$-diarylalkylmethylamines, ${ }^{4}$ methodologies employing additions to benzophenone-derived imines are few and far between. ${ }^{5}$ The scarcity of latter methodologies stems from remarkably low reactivity of bis-aromatic ketimines, making an arylation of benzophenone imines even more challenging.
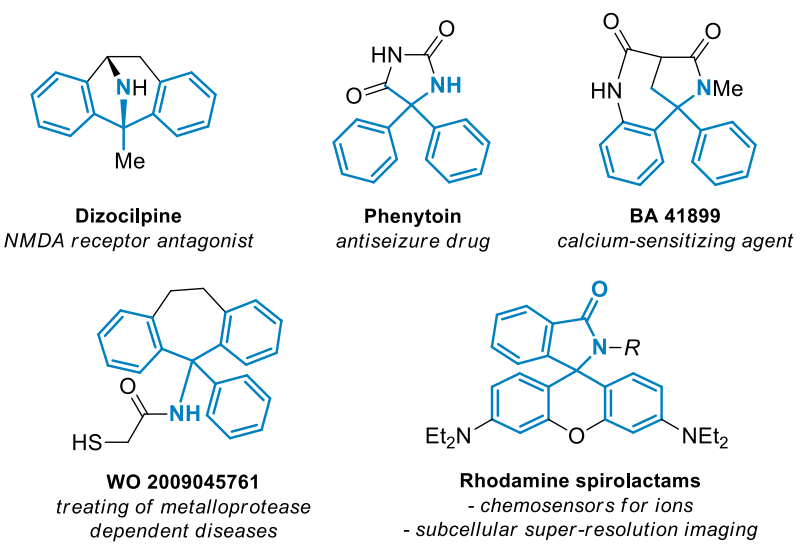

\section{Scheme 1. $\alpha$-Tertiary amines in bioactive molecules.}

A few notable examples of preparation of $\alpha$-triphenylmethylamines have appeared in the literature, though some of them within general studies on imine reactivity (Scheme 2). The Harutyunyan group $^{4 \mathrm{~b}}$ and the Bode group ${ }^{4 \mathrm{c}}$ reported 1,2-additions of Grignard reagents to activated benzophenone imines. During the development of radical coupling reactions between aryl halides and 2-azaallyl species, Walsh et al obtained $\alpha$-triphenylmethylamine motifs in low yields as side products. ${ }^{6}$ In rhodium-catalyzed reactions, a series of enantioenriched $\alpha$-triphenylmethylamines 
were prepared by employing cyclic $N$-sulfonyl benzophenone-derived imines as reactive electrophiles. ${ }^{7}$ This concept was extended to a series of palladium-catalyzed transformations, ${ }^{8}$ as well as to cyclic amides. ${ }^{9}$

- Addition of organometallic reagents
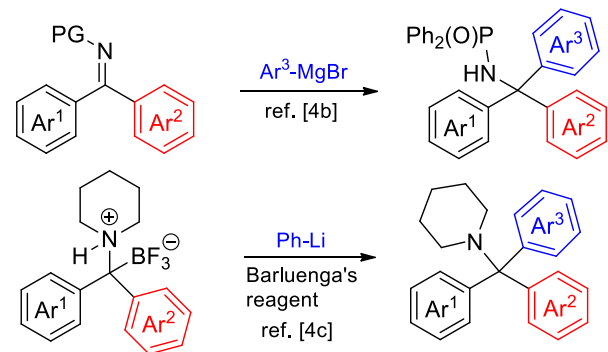

- Transition metal-catalyzed coupling

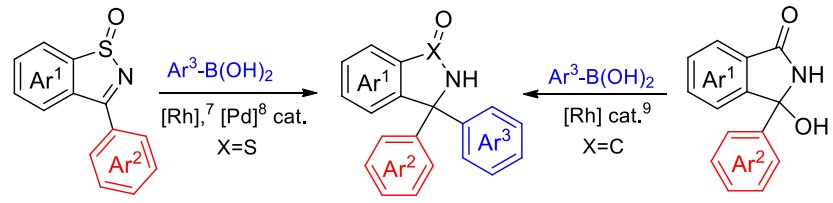

- This work: Brønsted acid-catalyzed formal Betti reaction

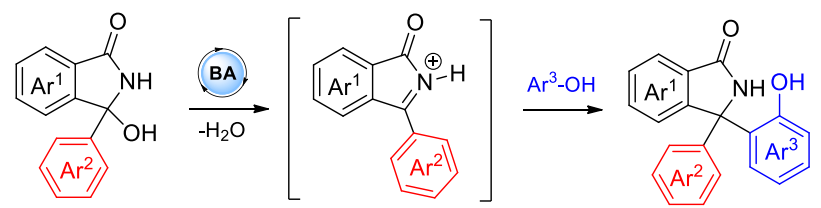

Scheme 2. Strategies towards $\alpha$-triphenylmethylamines.

In contrast to these elegant examples, to the best of our knowledge there are no reports on the synthesis of $\alpha$-triphenylmethylamines under organocatalytic conditions. Development of such protocols would add a new dimension to already existing methods, allowing more versatility in the preparation of these valuable structural units.

Herein, we report a Brønsted acid-catalyzed formal Betti reaction - a 1,2-addition of phenols to $N$-acyl benzophenone-ketimines, generated in situ from $\alpha^{N}$-hydroxy amides. The Betti reaction is a Mannich-type multicomponent reaction between aldehydes, primary amines, and naphthols for the synthesis of $\alpha$-secondary amines, so-called Betti bases. ${ }^{10}$ Although phenols can be used in the Betti reaction, 2-naphthols are preffered nucleophiles because of their higher reactivity in 
comparison to standard phenol derivatives, especially in rare examples describing the formation of $\alpha$-diarylalkylmethylamine products. ${ }^{11}$ In recent years, a number of methodologies were developed where reactive imine species were formed in situ from their stable precursors, 3hydroxyisoindolinones, and utilized in various transformations. ${ }^{12}$ Based on reported strategies, we reasoned that benzophenone-derived iminium species generated in this way might act as highly reactive electrophiles for poor nucleophiles, such as phenols, and enable 1,2-addition of aryl rings in a formal Betti reaction.

We started our investigations by combining 3-phenyl 3-hydroxyisoindolinone 32 with $p$ chlorophenol in the presence of various Brønsted and Lewis acids (Table 1).

Table 1. Screening of reaction conditions. ${ }^{\mathrm{a}}$
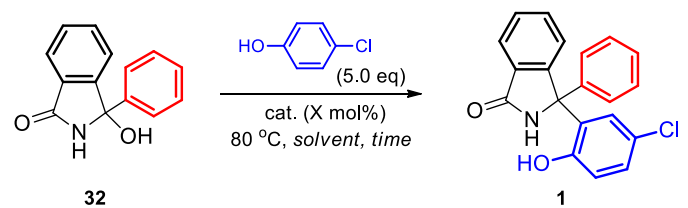

\begin{tabular}{|c|c|c|c|c|}
\hline Entry & Cat. (mol\%) & Solvent & Time (h) & Yield (\%) \\
\hline 1 & $p$ TsOH (20) & toluene & 16 & 77 \\
\hline 2 & PhCOOH (20) & toluene & 60 & 27 \\
\hline 3 & $\mathrm{AcOH}$ & toluene & 60 & 67 \\
\hline 4 & $\mathrm{H}_{2} \mathrm{SO}_{4}(20)$ & toluene & 10 & 50 \\
\hline 5 & $\mathrm{MsOH}(20)$ & toluene & 12 & 98 \\
\hline 6 & $\mathrm{BF}_{3} \times \mathrm{OEt}_{2}(20)$ & toluene & 48 & 43 \\
\hline 7 & $\mathrm{SnCl}_{2}(20)$ & toluene & 24 & 53 \\
\hline 8 & $\mathrm{Sc}(\mathrm{OTf})_{2}(20)$ & toluene & 24 & 90 \\
\hline 9 & $\mathrm{MsOH}(20)$ & $o$-xylene & 24 & 82 \\
\hline 10 & $\mathrm{MsOH}(20)$ & cyclohexane & 4 & 92 \\
\hline 11 & $\mathrm{MsOH}(20)$ & heptane & 5 & 70 \\
\hline 12 & MsOH (10) & cyclohexane & 16 & $89^{b}$ \\
\hline 13 & $\mathrm{MsOH}(5)$ & cyclohexane & 48 & $\operatorname{traces}^{\mathrm{b}}$ \\
\hline 14 & $\mathrm{MsOH}(10)$ & cyclohexane & 72 & $88^{\mathrm{b}, \mathrm{c}}$ \\
\hline
\end{tabular}

${ }^{a}$ Reactions were carried out on $0.2 \mathrm{mmol}$ scale. ${ }^{b} p$-Chlorophenol $(1.5 \mathrm{eq}) \cdot{ }^{c} 60^{\circ} \mathrm{C}$. 
Our initial attempt with $p$-toluenesulfonic acid in toluene at $80{ }^{\circ} \mathrm{C}$ led to the desired product $\mathbf{1}$ within 16 hours (77\% yield, entry 1). Employing carboxylic acids as catalysts substantially prolonged reaction time; after 60 hours, product 1 was isolated in 27\% (benzoic acid, entry 2) and $67 \%$ (acetic acid, entry 3) yield, respectively. By using catalytic amount of sulfuric acid, the reaction was completed within 10 hours, though decomposition products were observed along with the product (50\% yield, entry 4 ). On the other hand, methanesulfonic acid yielded product $\mathbf{1}$ in almost quantitative yield after 12 hours (entry 5).

Next, the catalytic efficiency of various Lewis acids was investigated. In general, reaction times were significantly prolonged. $\mathrm{BF}_{3} \times \mathrm{OEt}_{2}$ (43\% yield, 48 hours, entry 6) and $\mathrm{SnCl}_{2}$ (53\% yield, 24 hours, entry 7) successfully catalyzed the transformation in moderate yields, while substantially better yield was obtained by employing $\mathrm{Sc}(\mathrm{OTf})_{2}$ as catalyst (90\% yield, 24 hours, entry 8 ).

After identifying methanesulfonic acid as the catalyst of choice for the transformation, the influence of solvent, temperature, and catalyst/reagent loading was investigated. By conducting the reaction in $o$-xylene for 24 hours at $80{ }^{\circ} \mathrm{C}$, product $\mathbf{1}$ was isolated in $82 \%$ yield (entry 9 ). Significant improvements in terms of reaction times were observed when cyclohexane ( $92 \%$ yield, 4 hours, entry 10) and heptane (70\% yield, 5 hours, entry 11$)$ were used as solvents, respectively. The reaction maintained its effectiveness when the amount of $p$-chlorophenol was lowered to 1.5 eq, accompanied by 10 mol\% catalyst loading ( $89 \%$ yield, 16 hours, entry 12$)$. Further decrease of catalyst loading to $5 \mathrm{~mol} \%$ resulted only in traces of the product, even after 48 hours (entry 13 ). Finally, performing the reaction under entry 11 parameters at $60^{\circ} \mathrm{C}$ prolonged its time to 72 hours (entry 14). Hence, chosen reaction conditions for the formal Betti reaction include diarylketimine precursor (1.0 eq), phenol (1.5 eq) and methanesulfonic acid $(10 \mathrm{~mol} \%)$ in cyclohexane at $80{ }^{\circ} \mathrm{C}$. 
With the optimized reaction conditions in hand (in terms of ratio between the catalyst and reagent loading, and obtained yield), we turned our attention to investigate the substrate scope and reaction limitations (Table 2).

Table 2. Substrate scope I: Phenols. ${ }^{\text {a }}$
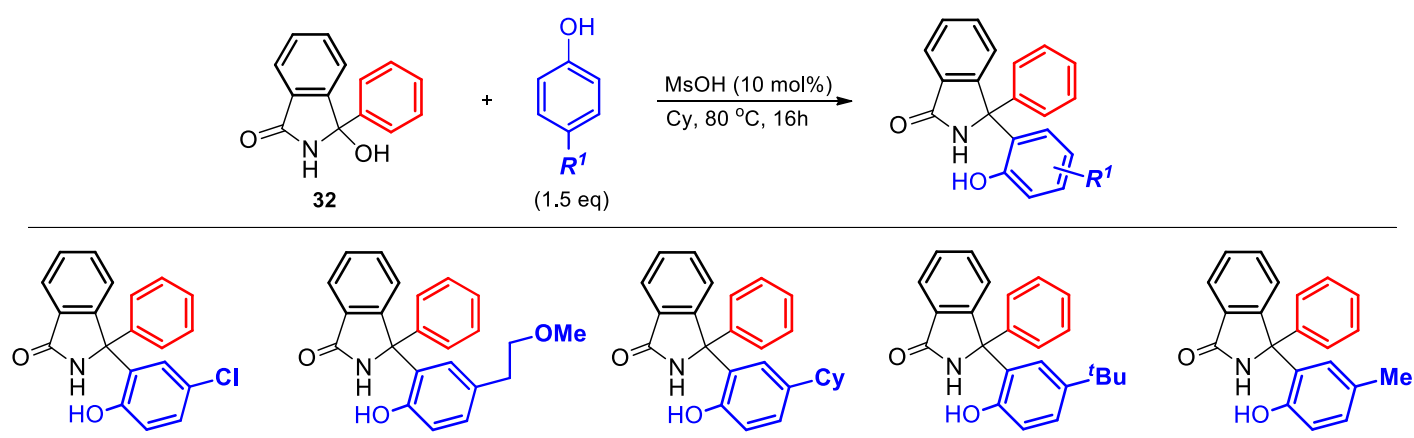

$$
1,89 \% \text { yield }
$$

2, $97 \%$ yield

$3,84 \%$ yield

4, 95\% yield

$5,77 \%$ yield
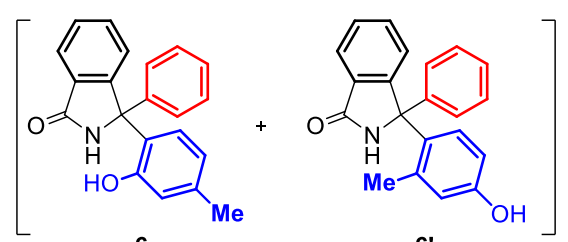

6
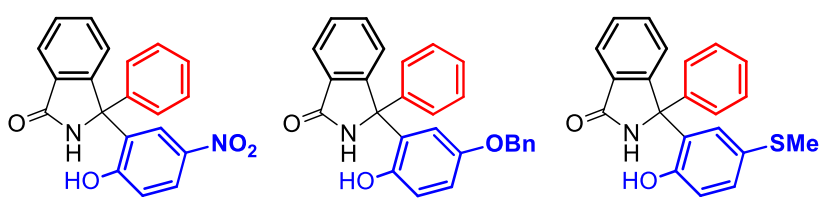

7, $84 \%$ yield

$8,82 \%$ yield

9, $88 \%$ yield (ortho $(\mathbf{6})$ vs para $\left.\left(6^{\prime}\right)=3: 1\right)^{\text {b }}$<smiles>O=C1NC(c2ccccc2)(c2cc(Oc3ccc(Br)cc3)ccc2O)c2ccccc21</smiles>
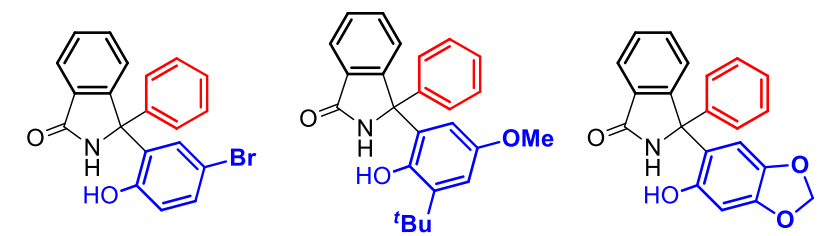

$12,81 \%$ yield $^{c}$

$13,89 \%$ yield $^{d}$

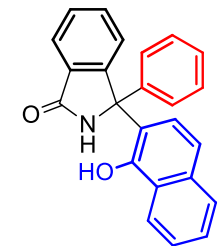

$14,95 \%$ yield $11,60 \%$ yield
(1g scale: $58 \%$ yield)

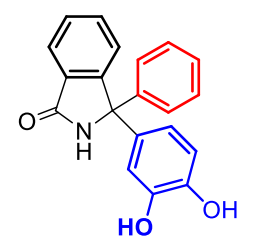

$15,75 \%$ yield

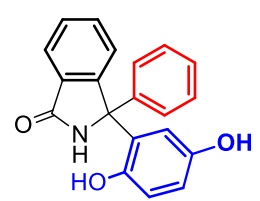

$16,34 \%$ yield

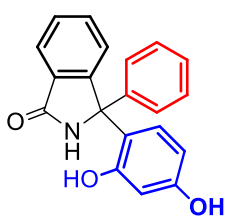

$17,50 \%$ yield

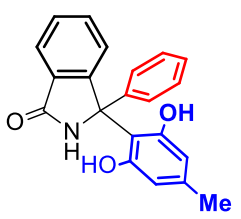

$18,50 \%$ yield

${ }^{a}$ Reactions were carried out on $0.2 \mathrm{mmol}$ scale. ${ }^{b}$ Inseparable mixture of ortho and para addition products with respect to hydroxy group. Ratio determined by ${ }^{1} \mathrm{H}$ NMR. ${ }^{c}$-(tert-butyl)-4-methoxyphenol (5.0 eq), $p$ TsOH (20 mol\%), $48 \mathrm{~h}$. ${ }^{d}$ Sesamol (1.5 eq), $p$ TsOH (20 mol\%), 16h.

The reaction maintained its effectiveness upon changing the halogen atom in para position of the phenol to alkyl groups (2-5). In order to investigate regioselectivity of the reaction, $m$-cresol was employed as a nucleophile. Under the same reaction conditions, products $\mathbf{6}$ and $\mathbf{6}^{\prime}$ were isolated in 
$81 \%$ yield as an inseparable mixture of ortho $(\mathbf{6})$ vs para $\left(\mathbf{6}^{\prime}\right)$ addition products (with respect to hydroxy group) in 3:1 ratio. The ratio between two regioisomers did not significantly change when the reaction was performed at $60{ }^{\circ} \mathrm{C}$, or at $100{ }^{\circ} \mathrm{C}$, respectively (see Supporting Information for details). Introduction of activating and deactivating groups in para position had little or no influence on product yield or the reaction course $(\mathbf{7}-\mathbf{1 0},>80 \%$ yield). In comparison to $p$ chlorophenol, the reaction with $p$-bromophenol resulted in significantly lower yield (11, 60\% yield), however, the reaction did not lose its effectiveness when performed on a 1.0 gram scale. Under the same conditions, the reaction with 2,4-disubstituted phenol afforded complex reaction mixture in which product 12 was not detected. The reason most likely lies in the cleavage of arylmethyl ethers with methanesulfonic acid under elevated temperatures. ${ }^{13}$ By switching the catalyst to $p$-toluenesulfonic acid and by increasing the amount of phenol to $5.0 \mathrm{eq}$, product $\mathbf{1 2}$ was obtained in $81 \%$ yield. Following the same reasoning, the reaction conditions used for the formation of $\mathbf{1 2}$ were also employed in the synthesis of $\mathbf{1 3}$, and sesamol succesfully reacted with 3-hydroxyisoindolinone $32(\mathbf{1 3}, 89 \%$ yield). Under the standard reaction conditions, the reaction with 1-naphthol resulted in almost quantitative yield of product 14. 
Reactions with different dihydroxybenzenes were investigated next. Employing catechol as a nucleophile resulted in addition through para position with respect to one of hydroxy groups, generating more favorable 1,2,5-trisubstituted product $\mathbf{1 5}$ in 75\% yield. In reactions with quinol and resorcinol, products 16 and 17 were isolated in low yields (34\% and 50\% yield, respectively). Since starting isoindolinone alcohol $\mathbf{3 2}$ was completely consumed in both reactions, this observation can be attributed to problems encountered during the isolation of products. By conducting the reaction with orcinol, the addition occured almost exclusively through carbon positioned between two hydroxy groups, with only traces of the other regioisomer visible in NMR spectra $(\mathbf{1 8}, 50 \%$ yield $)$.

Table 3. Substrate scope II: Imine precursors. ${ }^{a}$

Next, various imine precursors were submitted to the reaction with a range of phenol derivatives (Table 3). In general, introduction of different substituents in para and meta positions on the 3-aryl ring of 3hydroxyisoindolinone provided products in good to excellent yields, regardless of the type of phenol derivative used. The reaction was tolerant of chloro, methyl, and trifluoromethyl substituents on both meta positions of the 3-phenyl ring (19-21), as well as with para substitutions on 3-phenyl ring (22 and 23). Placement of 2-naphtyl
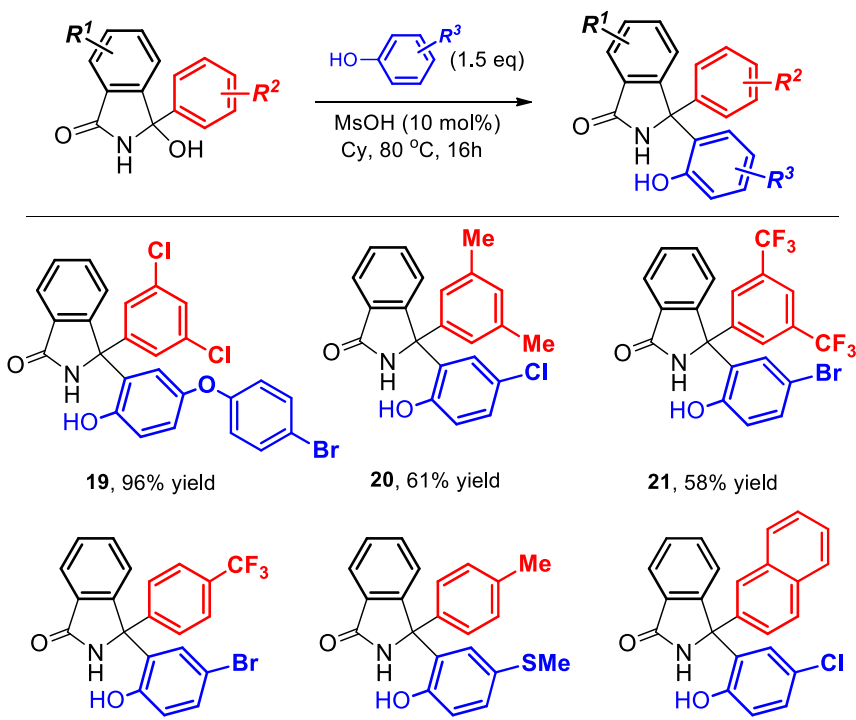

$$
22,72 \% \text { yield }
$$

$23,84 \%$ yield

$24,69 \%$ yield

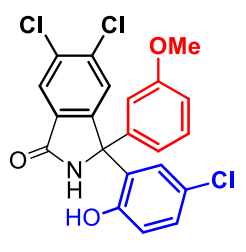

$25,79 \%$ yield

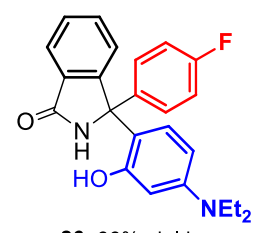

26, $66 \%$ yield

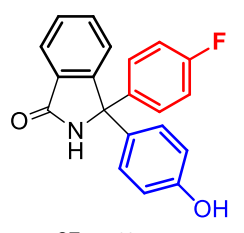

27, $96 \%$ yield

${ }^{a}$ Reactions were carried out on $0.2 \mathrm{mmol}$ scale unless otherwise noted (see Experimental section).

substituent also had no significant influence on the reaction outcome (24, 69\% yield), and similar trend was observed with imine precursor possessing substituted isoindolinone benzene ring (25, 
79\% yield). Employing $m$-diethylamino phenol as a nucleophile provided only one regioisomer $\mathbf{2 6}$ in 66\% yield, most likely because of increased steric hindrance around para position (with respect to hydroxy group). Finally, we investigated regioselectivity of the reaction when unsubstituted phenol was used, and only para regioisomer 27 was isolated in $96 \%$ yield.

On the other hand, the transformation did not proceed with ortho substituted 3-aryl substituents on the imine precursor. With mesityl substitution, only starting materials were retrieved, and the same result was obtained with 3-(o-methoxylphenyl) substituent. This limitation most likely stems from the increased steric hindrance around the reactive center.

In order to get a better insight on the reactivity of both reaction partners, experiments with $\mathrm{N}$ protected isoindolinone alcohol and $O$-protected phenol were performed (Scheme 3).

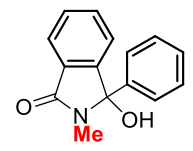

33

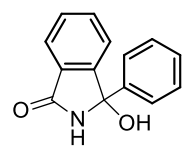

32

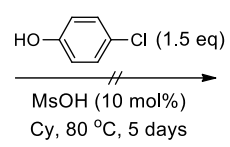

Cy, $80^{\circ} \mathrm{C}, 5$ days

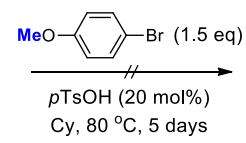

Cy, $80^{\circ} \mathrm{C}, 5$ days

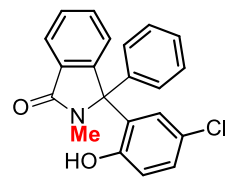

28

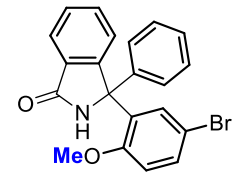

29

Scheme 3. Control experiments.

When $N$-methyl 3-hydroxyisoindolinone $\mathbf{3 3}$ and $p$-chlorophenol were submitted to reaction conditions, product 28 was not observed even after substantially prolonged reaction time. Likewise, in a reaction between isoindolinone alcohol $\mathbf{3 2}$ and $p$-bromoanisole catalyzed by $p$ toluenesulfonic acid, only starting materials were isolated from the reaction mixture. These experiments indicate that $\mathrm{NH}$ is required for the generation of reactive benzophenone iminium species, and that $\mathrm{OH}$ plays an important role in the nucleophilicity of the phenyl ring. 
Finally, we explored possibilities of synthetic utility of obtained products (Scheme 4). In addition to the successful scale-up reaction (11, Table 2), products of the formal Betti reaction were used in further transformations. Thus, $\mathrm{LiAlH}_{4}$-mediated reduction of amide group afforded products bearing $\alpha, \alpha$-disubstituted isoindoline core in very good and excellent yields $(\mathbf{3 0}, 84 \%$ yield, $\mathbf{3 1}$, $96 \%$ yield). Since one of the control experiments showed that the transformation does not proceed with anisole derivatives as nucleophiles, access to these compounds was demonstrated by employing standard $O$-alkylation (29, 65\% yield).
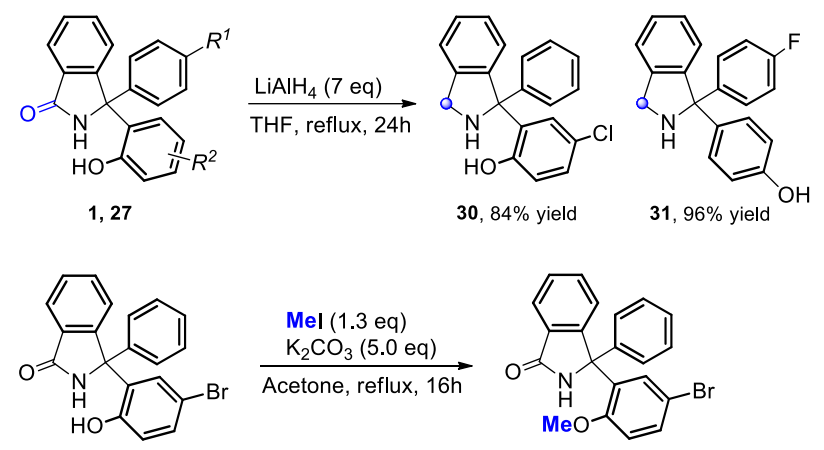

$30,84 \%$ yield
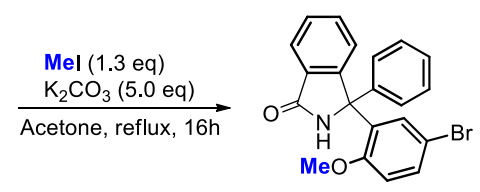

11

$29,65 \%$ yield

Scheme 4. Demonstration of synthetic utility.

In conclusion, we have developed a Brønsted acid-catalyzed formal Betti reaction between phenols and in situ generated diarylketimines from 3-aryl 3-hydroxyisoindolinones. The transformation proceeds smoothly with a broad range of phenols and benzophenone-derived imines to afford $\alpha$ triphenylmethylamines in good to excellent yields. Development of the stereoselective variant of this transformation is currently under way, and will be reported in due course.

\section{Experimental Section}

General Information. Chemicals and solvents were purchased from commercial suppliers and used as received. Unless otherwise noted, all compounds were prepared according to the General procedure. Flash column chromatography was carried out using silica gel (Merck, 40-63 $\mu \mathrm{m}$ 
particle size). NMR spectra were recorded on Bruker Avance 600 and $300 \mathrm{MHz}$ spectrometers, operating at 150.92 or $75.47 \mathrm{MHz}$ for ${ }^{13} \mathrm{C}$ and 600.13 or $300.13 \mathrm{MHz}$ for ${ }^{1} \mathrm{H}$ nuclei. Chemical shifts are quoted in ppm and are referenced to the residual nondeuterated solvent peak. If not otherwise noted, spectra were acquired at 298 K. Structural assignment were made with additional information from HMQC and HMBC experiments. Infrared spectra were recorded on a Varian UV/vis Cary 4000 spectrometer equipped with an attenuated total reflectance attachment with internal calibration. Absorption maxima $\left(v_{\max }\right)$ are reported in wavenumbers $\left(\mathrm{cm}^{-1}\right)$. High resolution mass spectrometry (HRMS) was performed on a 4800 Plus MALDI TOF/TOF Analyzer. Melting points were determined using an Electrothermal 9100 apparatus in open capillaries and are uncorrected. Substrates, 3-aryl 3-hydroxyisoindolinones 32-41 (see Supporting Information), were synthesized in high yields from readily available starting materials, by employing addition of a Grignard or organolithium reagent to phthalimide. ${ }^{14}$

General procedure. To a suspension of 3-aryl 3-hydroxyisoindolinone ( $0.2 \mathrm{mmol})$ in cyclohexane (2.0 mL) was added $\mathrm{MsOH}(19 \mathrm{mg}, 14 \mu \mathrm{L}, 0.02 \mathrm{mmol})$ at $25^{\circ} \mathrm{C}$. After stirring for $10 \mathrm{~min}$, phenol derivative $(0.3 \mathrm{mmol})$ was added, and the resulting reaction mixture was stirred in an oil bath for 16 hours at $80^{\circ} \mathrm{C}$. Full consumption of the starting material was confirmed by TLC, and the crude reaction mixture was directly purified by column chromatography on silica gel.

3-(5-Chloro-2-hydroxyphenyl)-3-phenylisoindolin-1-one (1). Colorless solid. Yield: 66 mg (89\%). Column chromatography eluents: dichloromethane-acetone 20:1. ${ }^{1} \mathrm{H}$ NMR (600 MHz, DMSO- $\left.d_{6}\right)$ $\delta 10.09(\mathrm{~s}, 1 \mathrm{H}), 9.10(\mathrm{~s}, 1 \mathrm{H}), 7.79(\mathrm{~d}, J=7.8 \mathrm{~Hz}, 1 \mathrm{H}), 7.72(\mathrm{~d}, J=7.2 \mathrm{~Hz}, 1 \mathrm{H}), 7.63(\mathrm{t}, J=7.8 \mathrm{~Hz}$, 1H), $7.53(\mathrm{t}, J=7.2 \mathrm{~Hz}, 1 \mathrm{H}), 7.28-7.26(\mathrm{~m}, 2 \mathrm{H}), 7.24-7.21(\mathrm{~m}, 2 \mathrm{H}), 7.17-7.14(\mathrm{~m}, 3 \mathrm{H}), 6.84(\mathrm{~d}, J$ $=8.4 \mathrm{~Hz}, 1 \mathrm{H}) .{ }^{13} \mathrm{C}\{1 \mathrm{H}\} \mathrm{NMR}\left(151 \mathrm{MHz}, \mathrm{DMSO}-d_{6}\right) \delta 168.2,154.3,149.2,143.0,132.0,131.1$, 130.2, 128.9, 128.6, 128.3, 126.9, 126.7, 125.3 (overlapping another signal), 123.4, 121.9, 118.0, 
68.7 - signals of the residual solvents (acetone and DCM) are visible in spectra. Mp 299.8-305.3 ${ }^{\circ} \mathrm{C} . v_{\max }$ (neat): $3370,3178,2955,2359,1676,1468,1316,1075,756,695,599,533 \mathrm{~cm}^{-1}$. HRMS (MALDI TOF) $m / z:[\mathrm{M}+\mathrm{H}]^{+}$calcd for $\mathrm{C}_{20} \mathrm{H}_{15} \mathrm{ClNO}_{2} 336.0713$; found 336.0726.

3-(2-Hydroxy-5-(2-methoxyethyl)phenyl)-3-phenylisoindolin-1-one (2). Colorless solid. Yield: 77 mg (97\%). Column chromatography eluents: dichloromethane-acetone 20:1. ${ }^{1} \mathrm{H}$ NMR (300 MHz, DMSO-d $d_{6} \delta 9.58(\mathrm{~s}, 1 \mathrm{H}), 8.90(\mathrm{~s}, 1 \mathrm{H}), 7.72(\mathrm{t}, J=6.6 \mathrm{~Hz}, 2 \mathrm{H}), 7.61(\mathrm{dt}, J=7.5,1.2 \mathrm{~Hz}, 1 \mathrm{H}), 7.51$ $(\mathrm{t}, J=7.8 \mathrm{~Hz}, 1 \mathrm{H}), 7.27-7.19(\mathrm{~m}, 3 \mathrm{H}), 7.15-7.13(\mathrm{~m}, 2 \mathrm{H}), 7.06-7.01(\mathrm{~m}, 2 \mathrm{H}), 6.76(\mathrm{~d}, J=8.1 \mathrm{~Hz}$, $1 \mathrm{H}), 3.41(\mathrm{t}, J=6.9 \mathrm{~Hz}, 2 \mathrm{H}), 3.18(\mathrm{~s}, 3 \mathrm{H}), 2.64(\mathrm{t}, J=6.9 \mathrm{~Hz}, 2 \mathrm{H}) .{ }^{13} \mathrm{C}\{1 \mathrm{H}\} \mathrm{NMR}(300 \mathrm{MHz}$, DMSO-d $\left.d_{6}\right) \delta 9.58(\mathrm{~s}, 1 \mathrm{H}), 8.90(\mathrm{~s}, 1 \mathrm{H}), 7.72(\mathrm{t}, J=6.6 \mathrm{~Hz}, 2 \mathrm{H}), 7.61(\mathrm{dt}, J=7.5,1.2 \mathrm{~Hz}, 1 \mathrm{H}), 7.51$ $(\mathrm{t}, J=7.8 \mathrm{~Hz}, 1 \mathrm{H}), 7.27-7.19(\mathrm{~m}, 3 \mathrm{H}), 7.15-7.13(\mathrm{~m}, 2 \mathrm{H}), 7.06-7.01(\mathrm{~m}, 2 \mathrm{H}), 6.76(\mathrm{~d}, J=8.1 \mathrm{~Hz}$, 1H), $3.41(\mathrm{t}, J=6.9 \mathrm{~Hz}, 2 \mathrm{H}), 3.18(\mathrm{~s}, 3 \mathrm{H}), 2.64(\mathrm{t}, J=6.9 \mathrm{~Hz}, 2 \mathrm{H}) . \mathrm{Mp} 237.1-240.9^{\circ} \mathrm{C} . v_{\max }($ neat): 3403, 2360, 1672, 1248, 1112, 699, $606 \mathrm{~cm}^{-1}$. HRMS (MALDI TOF) $\mathrm{m} / z:[\mathrm{M}+\mathrm{H}]^{+}$calcd for $\mathrm{C}_{23} \mathrm{H}_{22} \mathrm{NO}_{3} 360.1600$; found 360.1584 .

3-(5-Cyclohexyl-2-hydroxyphenyl)-3-phenylisoindolin-1-one (3). Colorless solid. Yield: $71 \mathrm{mg}$ (84\%). Column chromatography eluents: dichloromethane-acetone 20:1. ${ }^{1} \mathrm{H}$ NMR (300 MHz, DMSO-d $)_{6} \delta 9.52(\mathrm{~s}, 1 \mathrm{H}), 8.91(\mathrm{~s}, 1 \mathrm{H}), 7.74(\mathrm{~d}, J=12.6 \mathrm{~Hz}, 1 \mathrm{H}), 7.72(\mathrm{~d}, J=12.3 \mathrm{~Hz}, 1 \mathrm{H}), 7.62$ $(\mathrm{dt}, J=7.5,1.2 \mathrm{~Hz}, 1 \mathrm{H}), 7.50(\mathrm{dt}, J=7.5,0.9 \mathrm{~Hz}, 1 \mathrm{H}), 7.27-7.18(\mathrm{~m}, 3 \mathrm{H}), 7.14-7.12(\mathrm{~m}, 2 \mathrm{H}), 7.05-$ $7.00(\mathrm{~m}, 2 \mathrm{H}), 6.75(\mathrm{~d}, J=8.1 \mathrm{~Hz}, 1 \mathrm{H}), 2.35-2.28(\mathrm{~m}, 1 \mathrm{H}), 1.73-1.63(\mathrm{~m}, 5 \mathrm{H}), 1.32-1.12(\mathrm{~m}, 5 \mathrm{H})$ signals of the residual solvents (acetone and DCM) are visible in spectra. ${ }^{13} \mathrm{C}\{1 \mathrm{H}\}$ NMR (151 MHz, DMSO-d $) \delta 168.5,153.2,150.0,143.9,137.5,131.9,131.1,128.5,128.3,127.5,127.1$, 126.9, 125.6, 125.5, 125.3, 123.4, 116.4, 69.3, 43.1, 34.4, 26.4, 25.7. Mp 305.8-307.0 ${ }^{\circ} \mathrm{C} . v_{\max }$ (neat): 3424, 2924, 2360, 1662, 1428, 1359, 1246, 1117, 750, 696, $579 \mathrm{~cm}^{-1}$. HRMS (MALDI TOF) $m / z:[\mathrm{M}+\mathrm{H}]^{+}$calcd for $\mathrm{C}_{26} \mathrm{H}_{26} \mathrm{NO}_{2}$ 384.1964; found 384.1952. 
3-(5-(tert-Butyl)-2-hydroxyphenyl)-3-phenylisoindolin-1-one (4). Colorless solid. Yield: $75 \mathrm{mg}$ (95\%). Column chromatography eluents: dichloromethane-acetone 20:1. ${ }^{1} \mathrm{H}$ NMR (300 MHz, DMSO- $\left.d_{6}\right) \delta 9.52(\mathrm{~s}, 1 \mathrm{H}), 8.91(\mathrm{~s}, 1 \mathrm{H}), 7.75-7.70(\mathrm{~m}, 2 \mathrm{H}), 7.69(\mathrm{dt}, J=7.5,1.2 \mathrm{~Hz}, 1 \mathrm{H}), 7.51(\mathrm{t}$, $J=8.1 \mathrm{~Hz}, 1 \mathrm{H}), 7.28-7.13(\mathrm{~m}, 8 \mathrm{H}), 6.76(\mathrm{~d}, J=8.1 \mathrm{~Hz}, 1 \mathrm{H}), 1.16(\mathrm{~s}, 9 \mathrm{H}) .{ }^{13} \mathrm{C}\{1 \mathrm{H}\} \mathrm{NMR}(75$ MHz, DMSO- $\left.d_{6}\right) \delta 168.6,152.9,150.0,144.0,140.5,131.8,131.1,128.5,128.3,127.0,126.9$, 126.6, 125.6, 125.3, 124.1, 123.5, 116.0, 69.4, 34.0, 31.5. Mp 284.1-285.0 ${ }^{\circ} \mathrm{C} . v_{\max }$ (neat): 3407 , 2962, 2360, 1681, 1416, 1373, 1265, 1127, 823, 702, $608 \mathrm{~cm}^{-1}$. HRMS (MALDI TOF) $\mathrm{m} / \mathrm{z}:[\mathrm{M}+$ $\mathrm{H}]^{+}$calcd for $\mathrm{C}_{24} \mathrm{H}_{25} \mathrm{NO}_{2}$ 358.1807; found 358.1817.

3-(2-Hydroxy-5-methylphenyl)-3-phenylisoindolin-1-one (5). Colorless solid. Yield: $53 \mathrm{mg}$ (77\%). Column chromatography eluents: dichloromethane-acetone 50:1. ${ }^{1} \mathrm{H}$ NMR $\left(600 \mathrm{MHz}, \mathrm{DMSO}-d_{6}\right)$ $\delta 9.51(\mathrm{~s}, 1 \mathrm{H}), 8.88(\mathrm{~s}, 1 \mathrm{H}), 7.73(\mathrm{~d}, J=14.3 \mathrm{~Hz}, 1 \mathrm{H}), 7.71(\mathrm{~d}, J=14.1 \mathrm{~Hz}, 1 \mathrm{H}), 7.61(\mathrm{t}, J=15.0$ $\mathrm{Hz}, 1 \mathrm{H}), 7.50$ (t, $J=15.0 \mathrm{~Hz}, 1 \mathrm{H}), 7.26-7.23(\mathrm{~m}, 2 \mathrm{H}), 7.20-7.15$ (m, 3H), 7.00 (s, 1H), 6.97 (d, $J$ $=7.8 \mathrm{~Hz}, 1 \mathrm{H}), 6.73(\mathrm{~d}, J=7.8 \mathrm{~Hz}, 1 \mathrm{H}), 2.15(\mathrm{~s}, 3 \mathrm{H}) .{ }^{13} \mathrm{C}\{1 \mathrm{H}\} \mathrm{NMR}\left(151 \mathrm{MHz}\right.$, DMSO- $\left.d_{6}\right) \delta$ $168.2,152.9,149.8,143.9,131.7,131.1,129.5,128.3,128.1,127.7,127.5,126.72,126.67,125.5$, 125.2, 123.2, 116.3, 69.0, 20.4 - signals of the residual solvents (acetone and DCM) are visible in spectra. Mp 302.6-305.3 ${ }^{\circ} \mathrm{C} . v_{\max }$ (neat): 3401, 2360, 1673, 1418, 1369, 1249, 1059, 759, 697, 566 $\mathrm{cm}^{-1}$. HRMS (MALDI TOF) $m / z:[\mathrm{M}+\mathrm{H}]^{+}$calcd for $\mathrm{C}_{21} \mathrm{H}_{18} \mathrm{NO}_{2}$ 316.1338; found 316.1350 .

3-(2-Hydroxy-4-methylphenyl)-3-phenylisoindolin-1-one (6) and 3-(4-Hydroxy-2-methylphenyl)3-phenylisoindolin-1-one $\left(\mathbf{6}^{\prime}\right)$. Inseparable mixture of regioisomers 6 and $\mathbf{6}^{\prime}$ in in 3:1 relative ratio as colorless solid. Regioisomers and their ratio was determined on the basis of ${ }^{1} \mathrm{H}, \mathrm{HSQC}$ and HMBC NMR experiments (see Supporting Information). Yield: $56 \mathrm{mg}$ (81\%). Column chromatography eluents: dichloromethane-acetone 50:1. 6: ${ }^{1} \mathrm{H}$ NMR (600 MHz, DMSO- $\left.d_{6}\right) \delta 9.63$ (s, 1H), $8.86(\mathrm{~s}, 1 \mathrm{H}), 7.69(\mathrm{~d}, J=7.2 \mathrm{~Hz}, 1 \mathrm{H}), 7.66(\mathrm{~d}, J=7.2 \mathrm{~Hz}, 1 \mathrm{H}), 7.59$ (t, $J=7.2 \mathrm{~Hz}, 1 \mathrm{H})$, 
$7.49(\mathrm{t}, J=6.6 \mathrm{~Hz}, 1 \mathrm{H}), 7.32(\mathrm{~d}, J=4.2 \mathrm{~Hz}, 1 \mathrm{H}), 7.23(\mathrm{~d}, J=7.2 \mathrm{~Hz}, 2 \mathrm{H}), 7.17(\mathrm{~d}, J=7.2 \mathrm{~Hz}$, 2H), $7.02(\mathrm{~d}, J=7.8 \mathrm{~Hz}, 1 \mathrm{H}), 6.64(\mathrm{~s}, 1 \mathrm{H}), 6.57(\mathrm{~d}, J=9.0 \mathrm{~Hz}, 1 \mathrm{H}), 2.20(\mathrm{~s}, 3 \mathrm{H}) .{ }^{13} \mathrm{C}\{1 \mathrm{H}\} \mathrm{NMR}$ (151 MHz, DMSO-d6) $\delta 168.3,155.1,149.9,143.9,138.7,131.7,131.1,128.6,128.1,127.1$, $126.7,125.4,125.2,125.1,123.2,119.0,117.0,68.8,20.6$ - signals of the residual solvents (acetone and DCM) are visible in spectra. 6': ${ }^{1} \mathrm{H}$ NMR $\left(600 \mathrm{MHz}, \mathrm{DMSO}-d_{6}\right) \delta 9.40(\mathrm{~s}, 1 \mathrm{H}), 9.36$ (s, 1H), $7.45(\mathrm{t}, J=7.8 \mathrm{~Hz}, 2 \mathrm{H}), 6.72(\mathrm{~d}, J=8.4 \mathrm{~Hz}, 1 \mathrm{H}), 6.47(\mathrm{dd}, J=9.0,3.0 \mathrm{~Hz}, 1 \mathrm{H}), 1.76(\mathrm{~s}$, $3 \mathrm{H})$ - rest of the signals are overlapped by the signals of the major regioisomer. ${ }^{13} \mathrm{C}$ NMR $(151$ MHz, DMSO- $\left.d_{6}\right) \delta 169.1,156.9,150.8,144.5,138.5,131.9,130.7,129.0,125.3,125.3,125.1$, $123.3,119.4,111.8,70.4,30.7,21.4$ - rest of the signals are overlapped by the signals of the major regioisomer. $\operatorname{Mp} 256.3-257.3^{\circ} \mathrm{C} . v_{\max }$ (neat): 3410, 3050, 1672, 1240, $695 \mathrm{~cm}^{-1}$. HRMS (MALDI TOF) $m / z:[\mathrm{M}+\mathrm{H}]^{+}$calcd for $\mathrm{C}_{21} \mathrm{H}_{18} \mathrm{NO}_{2} 316.1338$; found 316.1332.

3-(2-Hydroxy-5-nitrophenyl)-3-phenylisoindolin-1-one (7). Yellow solid. Yield: $64 \mathrm{mg}$ (84\%). Column chromatography eluents: dichloromethane-acetone 10:1. ${ }^{1} \mathrm{H}$ NMR (600 MHz, DMSO- $\left.d_{6}\right)$ $\delta 11.53$ (brs, 1H), 9.33 (s, 1H), 8.13 (dd, $J=8.4,2.4 \mathrm{~Hz}, 1 \mathrm{H}), 8.12(\mathrm{~d}, J=3.0 \mathrm{~Hz}, 1 \mathrm{H}), 7.81(\mathrm{~d}, J$ $=7.8 \mathrm{~Hz}, 1 \mathrm{H}), 7.75(\mathrm{~d}, J=7.8 \mathrm{~Hz}, 1 \mathrm{H}), 7.67(\mathrm{dt}, J=7.8,0.6 \mathrm{~Hz}, 1 \mathrm{H}), 7.56(\mathrm{dt}, J=7.8,0.6 \mathrm{~Hz}$, 1H), 7.30-7.27 (m, 2H), 7.25-7.23 (m, 1H), 7.21-7.19 (m, 2H), $6.97(\mathrm{~d}, J=9.0 \mathrm{~Hz}, 1 \mathrm{H})$ - signal of the residual solvent (acetone) is visible in spectrum. ${ }^{13} \mathrm{C}\{1 \mathrm{H}\} \mathrm{NMR}\left(151 \mathrm{MHz}, \mathrm{DMSO}-d_{6}\right) \delta 168.4$, $148.9,142.6,138.6,132.2,131.2,129.3,128.9,128.4,127.1,126.0,125.2,125.1,123.5,123.3$, 116.8, 68.6 - one signal in aromatic region is overlapped. Mp 275.7-277.2 ${ }^{\circ} \mathrm{C} . v_{\max }$ (neat): 3432 , 3395, 2721, 2358, 1584, 1334, 1079, $695 \mathrm{~cm}^{-1}$. HRMS (MALDI TOF) $\mathrm{m} / z:[\mathrm{M}+\mathrm{H}]^{+}$calcd for $\mathrm{C}_{20} \mathrm{H}_{14} \mathrm{~N}_{2} \mathrm{O}_{4} 347.1032$; found 347.1047.

3-(5-(Benzyloxy)-2-hydroxyphenyl)-3-phenylisoindolin-1-one (8). Colorless solid. Yield: $73 \mathrm{mg}$ (82\%). Column chromatography eluents: dichloromethane-acetone 20:1. ${ }^{1} \mathrm{H}$ NMR (600 MHz, 
DMSO- $\left.d_{6}\right) \delta 9.34(\mathrm{~s}, 1 \mathrm{H}), 8.88(\mathrm{~s}, 1 \mathrm{H}), 7.71(\mathrm{~d}, J=11.0 \mathrm{~Hz}, 1 \mathrm{H}), 7.66(\mathrm{~d}, J=11.7 \mathrm{~Hz}, 1 \mathrm{H}), 7.60$ $(\mathrm{dt}, J=7.5,1.2 \mathrm{~Hz}, 1 \mathrm{H}), 7.51(\mathrm{dt}, J=7.2 \mathrm{~Hz}, 1.2 \mathrm{~Hz}, 1 \mathrm{H}), 7.36-7.14(\mathrm{~m}, 10 \mathrm{H}), 6.88-6.73(\mathrm{~m}, 3 \mathrm{H})$, $4.93(\mathrm{~s}, 2 \mathrm{H}) .{ }^{13} \mathrm{C}\{1 \mathrm{H}\}$ NMR $\left(151 \mathrm{MHz}, \mathrm{DMSO}-d_{6}\right) \delta 168.3,150.4,149.5,149.1,143.6,137.2$, 131.9, 131.0, 128.7, 128.41, 128.36, 128.2, 127.7 (two signals overlapping), 126.8, 125.4, 125.2, 123.3, 116.8, 115.0, 114.6, 69.8, 68.9. Mp 243.8-245.6 ${ }^{\circ} \mathrm{C} . v_{\max }$ (neat): $3396,2360,1674,1428$, 1210, 1025, 696, $593 \mathrm{~cm}^{-1}$. HRMS (MALDI TOF) $\mathrm{m} / z:[\mathrm{M}+\mathrm{H}]^{+}$calcd for $\mathrm{C}_{27} \mathrm{H}_{22} \mathrm{NO}_{3} 408.1600$; found 408.1586 .

3-(2-Hydroxy-5-(methylthio)phenyl)-3-phenylisoindolin-1-one (9). Colorless solid. Yield: $67 \mathrm{mg}$ (88\%). Column chromatography eluents: dichloromethane-acetone 20:1. ${ }^{1} \mathrm{H}$ NMR (600 MHz, DMSO- $\left.d_{6}\right) \delta 9.84(\mathrm{~s}, 1 \mathrm{H}), 9.02(\mathrm{~s}, 1 \mathrm{H}), 7.77(\mathrm{~d}, J=7.8 \mathrm{~Hz}, 1 \mathrm{H}), 7.71(\mathrm{~d}, J=7.2 \mathrm{~Hz}, 1 \mathrm{H}), 7.62(\mathrm{dt}$, $J=7.8,1.2 \mathrm{~Hz}, 1 \mathrm{H}), 7.52(\mathrm{~d}, J=7.2 \mathrm{~Hz}, 1 \mathrm{H}), 7.27-7.15(\mathrm{~m}, 7 \mathrm{H}), 6.83(\mathrm{~d}, J=8.4 \mathrm{~Hz}, 1 \mathrm{H}), 2.34(\mathrm{~s}$, $3 \mathrm{H})$ ) - signal of the residual solvent (acetone) is visible in spectrum. ${ }^{13} \mathrm{C}\{1 \mathrm{H}\} \mathrm{NMR}(151 \mathrm{MHz}$, DMSO- $\left.d_{6}\right) \delta 168.2,153.7,149.5,143.4,131.8,131.1,129.3,128.9,128.5,128.2,127.4,126.8$, 125.7, 125.4, 125.2, 123.3, 117.4, 68.9, 17.0. Mp 267.3-269.5 ${ }^{\circ} \mathrm{C} . v_{\max }$ (neat): $3406,2360,1683$, 1408, 1269, 696, $586 \mathrm{~cm}^{-1}$. HRMS (MALDI TOF) $\mathrm{m} / z:[\mathrm{M}+\mathrm{H}]^{+}$calcd for $\mathrm{C}_{21} \mathrm{H}_{18} \mathrm{NO}_{2} \mathrm{~S} 348.1058$ found 348.1068 .

3-(5-(4-Bromophenoxy)-2-hydroxyphenyl)-3-phenylisoindolin-1-one (10). Colorless solid. Yield: $86 \mathrm{mg}(83 \%)$. Column chromatography eluents: dichloromethane-acetone 20:1. ${ }^{1} \mathrm{H}$ NMR (300 MHz, DMSO-d $d_{6} \delta 9.80(\mathrm{~s}, 1 \mathrm{H}), 9.04(\mathrm{~s}, 1 \mathrm{H}), 7.75-7.69(\mathrm{~m}, 2 \mathrm{H}), 7.59(\mathrm{t}, J=7.5 \mathrm{~Hz}, 1 \mathrm{H}), 7.52-$ $7.46(\mathrm{~m}, 3 \mathrm{H}), 7.30-7.17(\mathrm{~m}, 5 \mathrm{H}), 6.89-6.84(\mathrm{~m}, 5 \mathrm{H}) .{ }^{13} \mathrm{C}\{1 \mathrm{H}\}$ NMR $\left(151 \mathrm{MHz}, \mathrm{DMSO}-d_{6}\right) \delta$ $168.2,157.4,152.0,149.4,146.7,143.3,132.6,131.9,131.1,129.5,128.5,128.2,126.9,125.3$, $125.2,123.3,120.4,119.1,117.4,113.9,68.8$ - one signal in aromatic region is overlapped. Mp 
$155.5-156.8^{\circ} \mathrm{C} . v_{\max }$ (neat): $3417,3058,2342,1670,1480,1219,697,581 \mathrm{~cm}^{-1}$. HRMS (MALDI TOF) $m / z:[\mathrm{M}+\mathrm{H}]^{+}$calcd for $\mathrm{C}_{26} \mathrm{H}_{19} \mathrm{BrNO}_{3} 472.0548$; found 472.0546 .

3-(5-Bromo-2-hydroxyphenyl)-3-phenylisoindolin-1-one (11). Colorless solid. Yield: $50 \mathrm{mg}$ (60\%). Gram scale reaction: 3-hydroxy-3-phenylisoindolin-1-one 32 (1.0 g, $4.44 \mathrm{mmol})$ afforded $0.97 \mathrm{~g}(58 \%)$ of the title compound. Column chromatography eluents: dichloromethane-acetone 10:1. ${ }^{1} \mathrm{H}$ NMR (600 MHz, DMSO-d6) $\delta 10.11(\mathrm{~s}, 1 \mathrm{H}), 9.11(\mathrm{~s}, 1 \mathrm{H}), 7.79(\mathrm{~d}, J=7.8 \mathrm{~Hz}, 1 \mathrm{H}), 7.71$ $(\mathrm{d}, J=7.2 \mathrm{~Hz}, 1 \mathrm{H}), 7.63(\mathrm{dt}, J=7.8,0.6 \mathrm{~Hz}, 1 \mathrm{H}), 7.53(\mathrm{dt}, J=7.2,0.6 \mathrm{~Hz}, 1 \mathrm{H}), 7.35(\mathrm{dd}, J=8.4$, 2.4 Hz, 1H), 7.28-7.26 (m, 3H), 7.23-7.21 (m, 1H), 7.16-7.15 (m, 2H), $6.79(\mathrm{~d}, \mathrm{~J}=9.0 \mathrm{~Hz}, 1 \mathrm{H})$. ${ }^{13} \mathrm{C}\{1 \mathrm{H}\}$ NMR (151 MHz, DMSO-d $) \delta 168.2,154.8,149.3,143.0,132.0,131.9,131.1,130.7$, 129.5, 128.7, 128.3, 127.0, 125.3, 125.2, 123.4, 118.6, 109.5, 68.6. Mp 299.8-305.3 ${ }^{\circ} \mathrm{C} . v_{\max }($ neat): 3167, 1682, 1217, 1289, 962, $707 \mathrm{~cm}^{-1}$. HRMS (MALDI TOF) $\mathrm{m} / z:[\mathrm{M}+\mathrm{H}]^{+}$calcd for $\mathrm{C}_{20} \mathrm{H}_{15} \mathrm{BrNO}_{2}$ 402.0106; found 402.0110.

3-(3-(tert-Butyl)-2-hydroxy-5-methoxyphenyl)-3-phenylisoindolin-1-one (12). To a suspension of 3-hydroxy-3-phenylisoindolin-1-one $32(50 \mathrm{mg}, 0.22 \mathrm{mmol})$ in cyclohexane $(2.0 \mathrm{~mL})$ was added p-TsOH (8.4 mg, $0.04 \mathrm{mmol})$ at $25^{\circ} \mathrm{C}$. After stirring for $10 \mathrm{~min}, 2$-(tert-butyl)-4-methoxyphenol (60 $\mathrm{mg}, 0.33 \mathrm{mmol}$ ) was added, and the resulting reaction mixture was stirred in an oil bath for 48 hours at $80^{\circ} \mathrm{C}$. Full consumption of the starting material was confirmed by TLC and direct flash column chromatography of the reaction mixture (DCM/acetone 10:1) afforded $69 \mathrm{mg}(81 \%)$ of the title compound as colorless solid. ${ }^{1} \mathrm{H}$ NMR $\left(600 \mathrm{MHz}, \mathrm{DMSO}-d_{6}\right) \delta 8.97(\mathrm{~s}, 1 \mathrm{H}), 7.72(\mathrm{~d}, J=7.2$ $\mathrm{Hz}, 1 \mathrm{H}), 7.61(\mathrm{dt}, J=7.21 .2 \mathrm{~Hz}, 1 \mathrm{H}), 7.52-7.48(\mathrm{~m}, 2 \mathrm{H}), 7.26-7.24(\mathrm{~m}, 2 \mathrm{H}), 7.20-7.16(\mathrm{~m}, 3 \mathrm{H})$, $6.81(\mathrm{~d}, J=3.0 \mathrm{~Hz}, 1 \mathrm{H}), 6.69(\mathrm{~s}, 1 \mathrm{H}), 6.44(\mathrm{~d}, J=3.0 \mathrm{~Hz}, 1 \mathrm{H}), 3.58(\mathrm{~s}, 3 \mathrm{H}), 1.31(\mathrm{~s}, 9 \mathrm{H}) .{ }^{13} \mathrm{C}\{1 \mathrm{H}\}$ NMR (151 MHz, DMSO- $\left.d_{6}\right) \delta 168.8,152.5,150.2,146.5,144.0,143.0,134.4,131.9,130.4,128.4$, 128.3, 126.7, 125.6, 124.4, 123.4, 112.0, 111.4, 69.5, 54.9, 34.8, 29.9. Mp 257.4-258.6 ${ }^{\circ} \mathrm{C} . v_{\max }$ 
(neat): 3479, 2947, 2360, 1687, 1198, 1053, $695 \mathrm{~cm}^{-1}$. HRMS (MALDI TOF) $m / z:[\mathrm{M}+\mathrm{H}]^{+}$calcd for $\mathrm{C}_{25} \mathrm{H}_{26} \mathrm{NO}_{3} 388.1913$; found 388.1913.

3-(6-Hydroxybenzo[d][1,3]dioxol-5-yl)-3-phenylisoindolin-1-one (13). To a suspension of 3hydroxy-3-phenylisoindolin-1-one $32(50 \mathrm{mg}, 0.22 \mathrm{mmol})$ in cyclohexane $(2.0 \mathrm{~mL})$ was added $p$ $\mathrm{TsOH}(8.4 \mathrm{mg}, 0.04 \mathrm{mmol})$ at $25^{\circ} \mathrm{C}$. After stirring for $10 \mathrm{~min}$, sesamol (46 mg, $0.33 \mathrm{mmol}, 1.5$ eq) was added and the resulting reaction mixture was stirred in an oil bath for 16 hours at $80{ }^{\circ} \mathrm{C}$. Full consumption of the starting material was confirmed by TLC and direct flash column chromatography of the reaction mixture (DCM/acetone 10:1) afforded $68 \mathrm{mg}(89 \%)$ of the title compound as colorless solid. ${ }^{1} \mathrm{H}$ NMR $\left(300 \mathrm{MHz}, \mathrm{DMSO}-d_{6}\right) \delta 9.53(\mathrm{~s}, 1 \mathrm{H}), 8.87(\mathrm{~s}, 1 \mathrm{H}), 7.68(\mathrm{~d}$, $J=8.1 \mathrm{H}, 2 \mathrm{H}), 7.60(\mathrm{t}, J=7.2 \mathrm{~Hz}, 1 \mathrm{H}), 7.50(\mathrm{t}, J=7.2 \mathrm{~Hz}, 1 \mathrm{H}), 7.25-7.18(\mathrm{~m}, 5 \mathrm{H}), 6.65(\mathrm{~s}, 1 \mathrm{H})$, $6.45(\mathrm{~s}, 1 \mathrm{H}), 5.91(\mathrm{~d}, J=3.9 \mathrm{~Hz}, 2 \mathrm{H}) ;{ }^{13} \mathrm{C}\{1 \mathrm{H}\} \mathrm{NMR}\left(75 \mathrm{MHz}, \mathrm{DMSO}-d_{6}\right) \delta 168.2,150.4,149.9$, 147.2, 144.0, 139.0, 131.8, 131.0, 128.3, 128.1, 126.7, 125.4, 125.2, 123.3, 119.8, 107.1, 101.0, 98.6, 68.8; Mp 192.6-194.9 ${ }^{\circ} \mathrm{C} . v_{\max }$ (neat): 3246, 1681, 1439, 1183, 831, 700, 557; HRMS (MALDI-TOF) m/z: [M+H $]^{+}$calcd. for $\mathrm{C}_{21} \mathrm{H}_{16} \mathrm{NO}_{4}$ 346.1079; found 346.1080.

3-(1-Hydroxynaphthalen-2-yl)-3-phenylisoindolin-1-one (14). Colorless solid. Yield: $76 \mathrm{mg}$ (95\%). Column chromatography eluents: dichloromethane-acetone 5:1. ${ }^{1} \mathrm{H}$ NMR (600 MHz, DMSO-d $\left.d_{6}\right) \delta 9.77(\mathrm{~s}, 1 \mathrm{H}), 9.13(\mathrm{~s}, 1 \mathrm{H}), 8.21(\mathrm{~d}, J=8.4 \mathrm{~Hz}, 1 \mathrm{H}), 7.84(\mathrm{~d}, J=8.4 \mathrm{~Hz}, 1 \mathrm{H}), 7.79(\mathrm{~d}$, $J=7.8 \mathrm{~Hz}, 1 \mathrm{H}), 7.73(\mathrm{~d}, J=7.2 \mathrm{~Hz}, 1 \mathrm{H}), 7.63(\mathrm{t}, J=7.8 \mathrm{~Hz}, 1 \mathrm{H}), 7.52(\mathrm{t}, J=7.8 \mathrm{~Hz}, 1 \mathrm{H}), 7.49$ $7.44(\mathrm{~m}, 2 \mathrm{H}), 7.39(\mathrm{~d}, J=8.4 \mathrm{~Hz}, 1 \mathrm{H}), 7.36(\mathrm{~d}, J=8.4 \mathrm{~Hz}, 1 \mathrm{H}), 7.28-7.26(\mathrm{~m}, 2 \mathrm{H}), 7.23-7.20(\mathrm{~m}$, $3 \mathrm{H}) ;{ }^{13} \mathrm{C}\{1 \mathrm{H}\}$ NMR $\left(151 \mathrm{MHz}, \mathrm{DMSO}-d_{6}\right) \delta 168.5,150.8,150.2,144.6,134.1,131.9,131.2$, $128.4,128.3,127.8,126.8,126.3,125.5,125.4,125.22,125.18,125.16,123.6,123.3,121.9,118.8$, 69.4; Mp 258.4-259.1 ${ }^{\circ} \mathrm{C} . v_{\max }$ (neat): 3446, 2359, 1673, 1349, 1195, 750, $580 \mathrm{~cm}^{-1}$; HRMS (ESITOF) m/z: $[\mathrm{M}+\mathrm{H}]^{+}$calcd. for $\mathrm{C}_{24} \mathrm{H}_{18} \mathrm{NO}_{2} 352.1338$; found 352.1331. 
3-(3,4-Dihydroxyphenyl)-3-phenylisoindolin-1-one (15). Colorless solid. Yield: 56 mg (75 \%). Column chromatography eluents: dichloromethane-acetone 10:1. ${ }^{1} \mathrm{H}$ NMR (600 MHz, DMSO- $\left.d_{6}\right)$ $\delta 9.56(\mathrm{~s}, 1 \mathrm{H}), 8.91(\mathrm{brs}, 2 \mathrm{H}), 7.68(\mathrm{~d}, J=7.2 \mathrm{~Hz}, 1 \mathrm{H}), 7.61(\mathrm{t}, J=7.2 \mathrm{~Hz}, 1 \mathrm{H}), 7.55(\mathrm{~d}, J=7.8 \mathrm{~Hz}$, $1 \mathrm{H}), 7.49(\mathrm{t}, J=7.2 \mathrm{~Hz}, 1 \mathrm{H}), 7.34-7.26(\mathrm{~m}, 5 \mathrm{H}), 6.64(\mathrm{~d}, J=7.8 \mathrm{~Hz}, 1 \mathrm{H}), 6.61(\mathrm{~d}, J=2.4 \mathrm{~Hz}, 1 \mathrm{H})$, $6.44(\mathrm{dd}, J=8.4,2.4 \mathrm{~Hz}, 1 \mathrm{H}) ;{ }^{13} \mathrm{C}\{1 \mathrm{H}\} \mathrm{NMR}\left(151 \mathrm{MHz}, \mathrm{DMSO}-d_{6}\right) \delta 168.3,150.4,144.9,144.7$, 143.6, 134.0, 131.8, 131.1, 128.2, 127.3, 126.9, 124.7, 123.1, 118.0, 115.1, 114.8, 79.2, 69.8; Mp 162.2-163.0 ${ }^{\circ} \mathrm{C} . v_{\max }$ (neat): $3522,3354,3039,1655,1283,1115,748,450 \mathrm{~cm}^{-1}$. HRMS (MALDITOF) $\mathrm{m} / \mathrm{z}$ : $[\mathrm{M}+\mathrm{H}]^{+}$calcd. for $\mathrm{C}_{20} \mathrm{H}_{16} \mathrm{NO}_{3}$ 318.1130; found 318.1134. Regiochemistry of the reaction was confirmed on the basis of 2D NMR spectra (see Supporting Information for details). 3-(2,5-Dihydroxyphenyl)-3-phenylisoindolin-1-one (16). Colorless solid. Yield: $24 \mathrm{mg}$ (34\%). Column chromatography eluents: dichloromethane-acetone $3: 1 .{ }^{1} \mathrm{H}$ NMR (300 MHz, DMSO- $\left.d_{6}\right)$ $\delta 9.02(\mathrm{~s}, 1 \mathrm{H}), 8.80(\mathrm{~s}, 1 \mathrm{H}), 8.75(\mathrm{~s}, 1 \mathrm{H}), 7.72-7.66(\mathrm{~m}, 2 \mathrm{H}), 7.62(\mathrm{t}, J=6.3 \mathrm{~Hz}, 1 \mathrm{H}), 7.51(\mathrm{t}, J=$ $7.2 \mathrm{~Hz}, 1 \mathrm{H}), 7.28-7.15(\mathrm{~m}, 5 \mathrm{H}), 6.67-6.55(\mathrm{~m}, 3 \mathrm{H}) ;{ }^{13} \mathrm{C}\{1 \mathrm{H}\}$ NMR $\left(75 \mathrm{MHz}, \mathrm{DMSO}-d_{6}\right) \delta 168.3$, $149.8,149.2,147.5,143.8,131.7,131.0,128.4,128.3,128.1,126.7,125.5,125.2,123.2,117.0$ 115.2, 114.5, 68.9; Mp 238.4-239.4 ${ }^{\circ} \mathrm{C} . v_{\max }$ (neat): 3203, 1650, 1424, 1247, 1212, 1112, 695, 589 $\mathrm{cm}^{-1}$. HRMS (MALDI-TOF) m/z: [M+H] $]^{+}$calcd. for $\mathrm{C}_{20} \mathrm{H}_{16} \mathrm{NO}_{3}$ 318.1130; found 318.1125.

3-(2,4-Dihydroxyphenyl)-3-phenylisoindolin-1-one (17). Colorless solid. Yield: $36 \mathrm{mg}$ (50 \%). Column chromatography eluents: dichloromethane-acetone $3: 1 .{ }^{1} \mathrm{H}$ NMR (600 MHz, DMSO- $\left.d_{6}\right)$ $\delta 9.58(\mathrm{~s}, 1 \mathrm{H}), 9.34(\mathrm{~s}, 1 \mathrm{H}), 8.77(\mathrm{~s}, 1 \mathrm{H}), 7.68(\mathrm{t}, J=7.5 \mathrm{~Hz}, 1 \mathrm{H}), 7.62-7.55(\mathrm{~m}, 2 \mathrm{H}), 7.50-7.45$ (m, 1H), 7.27-7.16 (m, 5H), $6.89(\mathrm{~d}, J=8.4 \mathrm{~Hz}, 1 \mathrm{H}), 6.31(\mathrm{~d}, J=2.4 \mathrm{~Hz}, 1 \mathrm{H}), 6.16(\mathrm{dd}, J=8.7$, $2.4 \mathrm{~Hz}, 1 \mathrm{H}) ;{ }^{13} \mathrm{C}\{1 \mathrm{H}\} \mathrm{NMR}\left(75 \mathrm{MHz}, \mathrm{DMSO}-d_{6}\right) \delta 168.3,158.2,156.2,150.2,144.3,131.6$, $131.1,128.1,127.9,126.6,125.4,125.2,123.2,118.8,105.1,103.6,79.2,68.7 ;$ Mp 287.8-289.0 ${ }^{\circ} \mathrm{C} . v_{\max }$ (neat): $3204,1651,1424,1210,1132,1112,901,695 \mathrm{~cm}^{-1}$. HRMS (MALDI-TOF) m/z: 
$[\mathrm{M}+\mathrm{H}]^{+}$calcd. for $\mathrm{C}_{20} \mathrm{H}_{16} \mathrm{NO}_{3} 318.1130$; found 318.1128. Regiochemistry of the reaction was confirmed on the basis of 2D NMR spectra (see Supporting Information for details).

3-(2,6-Dihydroxy-4-methylphenyl)-3-phenylisoindolin-1-one (18). Colorless solid. Yield: $36 \mathrm{mg}$ (50\%). Column chromatography eluents: dichloromethane-acetone 5:1. ${ }^{1} \mathrm{H}$ NMR (600 MHz, DMSO-d $) \delta 9.20(\mathrm{~s}, 2 \mathrm{H}), 8.46(\mathrm{~s}, 1 \mathrm{H}), 7.55(\mathrm{~d}, J=7.2 \mathrm{~Hz}, 1 \mathrm{H}), 7.51(\mathrm{~d}, J=7.8 \mathrm{~Hz}, 1 \mathrm{H}), 7.37(\mathrm{t}$, $J=8.4 \mathrm{~Hz}, 1 \mathrm{H}), 7.32(\mathrm{t}, J=6.6 \mathrm{~Hz}, 1 \mathrm{H}), 7.23-7.19(\mathrm{~m}, 4 \mathrm{H}), 7.14-7.10(\mathrm{~m}, 1 \mathrm{H}), 6.07(\mathrm{~s}, 2 \mathrm{H}), 2.08$ $(\mathrm{s}, 3 \mathrm{H}) ;{ }^{13} \mathrm{C}\{1 \mathrm{H}\} \mathrm{NMR}\left(151 \mathrm{MHz}, \mathrm{DMSO}-d_{6}\right) \delta 168.7,156.3,152.1,146.7,137.9,130.0,129.9$, $128.0,127.9,126.9,125.9,125.4,124.1,121.6,111.4,108.2,79.1,67.9,20.61 ;$ Mp 165.1-165.3 ${ }^{\circ}$ C. $v_{\max }$ (neat): 3169, 2360, 1651, 1501, 1420, 1059, 694, $567 \mathrm{~cm}^{-1}$. HRMS (MALDI-TOF) m/z: $[\mathrm{M}+\mathrm{H}]^{+}$calcd. for $\mathrm{C}_{21} \mathrm{H}_{18} \mathrm{NO}_{3} 332.1287$; found 332.1277. Regiochemistry of the reaction was confirmed on the basis of 2D NMR spectra (see Supporting Information for details).

3-(5-(4-Bromophenoxy)-2-hydroxyphenyl)-3-(3,5-dichlorophenyl)isoindolin-1-one (19).

Colorless solid. Yield: 114 mg (96\%). Column chromatography eluents: dichloromethane-acetone 5:1. ${ }^{1} \mathrm{H}$ NMR (600 MHz, DMSO-d6) $\delta 9.95(\mathrm{~s}, 1 \mathrm{H}), 9.26(\mathrm{~s}, 1 \mathrm{H}), 7.85(\mathrm{~d}, J=7.8 \mathrm{~Hz}, 1 \mathrm{H}), 7.72(\mathrm{~d}$, $J=7.2 \mathrm{~Hz}, 1 \mathrm{H}), 7.64(\mathrm{dt}, J=7.2,1.2 \mathrm{~Hz}, 1 \mathrm{H}), 7.54(\mathrm{t}, J=7.2 \mathrm{~Hz}, 1 \mathrm{H}), 7.50(\mathrm{t}, J=1.8 \mathrm{~Hz}, 1 \mathrm{H})$, 7.48-7.46 (m, 2H), $7.161(\mathrm{~s}, 1 \mathrm{H}), 7.158(\mathrm{~s}, 1 \mathrm{H}), 6.95-6.85(\mathrm{~m}, 5 \mathrm{H})$ - signal of the residual solvent (acetone) is visible in spectrum. ${ }^{13} \mathrm{C}\{1 \mathrm{H}\}$ NMR (151 MHz, DMSO-d6) $\delta 168.1,157.1,151.7$, $148.04,147.97,147.0,134.0,132.6,132.4,130.9,129.1,128.4,126.8,125.1,124.1,123.6,120.8$, 119.3, 118.7, 117.6, 114.1, 68.0. Mp 287.8-288.2 ${ }^{\circ} \mathrm{C} . v_{\max }$ (neat): $3186,2360,1671,1223,576 \mathrm{~cm}^{-}$ 1. HRMS (MALDI TOF) $\mathrm{m} / z:[\mathrm{M}+\mathrm{H}]^{+}$calcd for $\mathrm{C}_{26} \mathrm{H}_{17} \mathrm{BrCl}_{2} \mathrm{NO}_{3}$ 539.6769; found 539.6768.

3-(5-Chloro-2-hydroxyphenyl)-3-(3,5-dimethylphenyl)isoindolin-1-one (20). Colorless solid. Yield: $48 \mathrm{mg}(61 \%)$. Column chromatography eluents: dichloromethane-acetone 5:1. ${ }^{1} \mathrm{H}$ NMR $\left(600 \mathrm{MHz}, \mathrm{DMSO}-d_{6}\right) \delta 10.06(\mathrm{~s}, 1 \mathrm{H}), 8.97(\mathrm{~s}, 1 \mathrm{H}), 7.77(\mathrm{~d}, J=7.8 \mathrm{~Hz}, 1 \mathrm{H}), 7.70(\mathrm{~d}, J=7.2 \mathrm{~Hz}$, 
1H), $7.63(\mathrm{dt}, J=7.8,1.2 \mathrm{~Hz}, 1 \mathrm{H}), 7.52(\mathrm{t}, J=7.2 \mathrm{~Hz}, 1 \mathrm{H}), 7.23(\mathrm{dd}, J=8.4,2.4 \mathrm{~Hz}, 1 \mathrm{H}), 7.10(\mathrm{~d}$, $J=2.4 \mathrm{~Hz}, 1 \mathrm{H}), 6.85(\mathrm{~s}, 1 \mathrm{H}), 6.83(\mathrm{~d}, J=8.4 \mathrm{~Hz}, 1 \mathrm{H}), 6.78(\mathrm{~s}, 2 \mathrm{H}), 2.17(\mathrm{~s}, 6 \mathrm{H}) .{ }^{13} \mathrm{C}\{1 \mathrm{H}\} \mathrm{NMR}$ (75 MHz, DMSO-d6) $\delta 168.3,154.4,149.3,143.0,137.2,132.0,131.1,130.4,128.9,128.6,128.5$, $126.8,125.3,123.4,122.9,121.9,118.0,68.6,21.1 . M p 287.3-289.2{ }^{\circ}$ C. $v_{\max }($ neat): 3066,1675 , 1269, $695 \mathrm{~cm}^{-1}$. HRMS (MALDI TOF) $\mathrm{m} / z$ : $[\mathrm{M}+\mathrm{H}]^{+}$calcd for $\mathrm{C}_{22} \mathrm{H}_{19} \mathrm{ClNO}_{2}$ 364.1104; found 364.1088 .

3-(3,5-Bis(trifluoromethyl)phenyl)-3-(5-bromo-2-hydroxyphenyl)isoindolin-1-one (21). Colorless solid. 3-(3,5-bis(trifluoromethyl)phenyl)-3-hydroxyisoindolin-1-one 36 (20 mg, $0.055 \mathrm{mmol})$ afforded $16 \mathrm{mg}(58 \%)$ of the title compound. Column chromatography eluents: dichloromethane-acetone 10:1. ${ }^{1} \mathrm{H}$ NMR (600 MHz, DMSO- $\left.d_{6}\right) \delta 10.34$ (brs, $\left.1 \mathrm{H}\right), 9.49$ (s, $\left.1 \mathrm{H}\right)$, $8.03(\mathrm{~s}, 1 \mathrm{H}), 7.93(\mathrm{~d}, J=7.8 \mathrm{~Hz}, 1 \mathrm{H}), 7.75(\mathrm{~d}, J=7.2 \mathrm{~Hz}, 1 \mathrm{H}), 7.74(\mathrm{~s}, 2 \mathrm{H}), 7.69$ (t, $J=7.2 \mathrm{~Hz}$, 1H), $7.59(\mathrm{t}, J=7.2 \mathrm{~Hz}, 1 \mathrm{H}), 7.38(\mathrm{dd}, J=8.4,2.4 \mathrm{~Hz}, 1 \mathrm{H}), 7.27(\mathrm{~d}, J=2.4 \mathrm{~Hz}, 1 \mathrm{H}), 6.78(\mathrm{~d}, J=$ $8.4 \mathrm{~Hz}, 1 \mathrm{H})$ - signal of the residual solvent (acetone) is visible in spectrum. ${ }^{13} \mathrm{C}\{1 \mathrm{H}\} \mathrm{NMR}(151$ MHz, DMSO-d6) $\delta 168.4,148.0,147.2,133.1,133.0,131.3,130.6(\mathrm{q}, J=32.9 \mathrm{~Hz}), 129.9,129.8$, 129.6, 126.1, 125.3, 124.4, 124.2, 122.6, 121.5, 119.0, 110.1, 68.3. Mp 298.5-299.7 ${ }^{\circ} \mathrm{C} . v_{\max }$ (neat): 3709, 3042, 2360, 1660, 1276, $681 \mathrm{~cm}^{-1}$. HRMS (MALDI TOF) $m / z:[\mathrm{M}+\mathrm{H}]^{+}$calcd for $\mathrm{C}_{22} \mathrm{H}_{13} \mathrm{BrF}_{6} \mathrm{NO}_{2}$ 516.0034; found 516.0054.

3-(5-Bromo-2-hydroxyphenyl)-3-(4-(trifluoromethyl)phenyl)isoindolin-1-one (22). Colorless solid. Yield: $71 \mathrm{mg}$ (72\%). Column chromatography eluents: dichloromethane-acetone 5:1. ${ }^{1} \mathrm{H}$ NMR (600 MHz, DMSO-d $) \delta 10.21(\mathrm{~s}, 1 \mathrm{H}), 9.27(\mathrm{~s}, 1 \mathrm{H}), 7.82(\mathrm{~d}, J=7.2 \mathrm{~Hz}, 1 \mathrm{H}), 7.75(\mathrm{~d}, J=$ 7.2 Hz, 1H), 7.68-7.65 (m, 3H), 7.57 (t, $J=7.8 \mathrm{~Hz}, 1 \mathrm{H}), 7.39-7.36(\mathrm{~m}, 3 \mathrm{H}), 7.27(\mathrm{~d}, J=2.4 \mathrm{~Hz}$, $1 \mathrm{H}), 6.81(\mathrm{~d}, J=8.4 \mathrm{~Hz}, 1 \mathrm{H})$ - signal of the residual solvent (acetone) is visible in spectrum. ${ }^{13} \mathrm{C}\{1 \mathrm{H}\} \mathrm{NMR}\left(151 \mathrm{MHz}, \mathrm{DMSO}-d_{6}\right) \delta 168.2,154.6,148.4,147.9,132.4,132.2,131.0,130.2$, 
$129.2,129.0,127.5(\mathrm{q}, J=31.7 \mathrm{~Hz}), 126.0,125.32,125.29,125.1,123.6,118.7,109.7,68.3 . \mathrm{Mp}$ 198.0-199. $6^{\circ} \mathrm{C} . v_{\max }$ (neat): $3184,2361,1668,1229,577 \mathrm{~cm}^{-1}$. HRMS (MALDI TOF) $\mathrm{m} / z:[\mathrm{M}+$ $\mathrm{H}]^{+}$calcd for $\mathrm{C}_{21} \mathrm{H}_{14} \mathrm{BrF}_{3} \mathrm{NO}_{2} 448.0160$; found 448.0150 .

3-(2-Hydroxy-5-(methylthio)phenyl)-3-(p-tolyl)isoindolin-1-one (23). Colorless solid. Yield: 66 mg (84\%). Column chromatography eluents: dichloromethane-acetone 5:1. ${ }^{1} \mathrm{H}$ NMR (600 MHz, DMSO- $\left.d_{6}\right) \delta 9.80(\mathrm{~s}, 1 \mathrm{H}), 8.95(\mathrm{~s}, 1 \mathrm{H}), 7.75(\mathrm{~d}, J=7.8 \mathrm{~Hz}, 1 \mathrm{H}), 7.70(\mathrm{~d}, J=7.2 \mathrm{~Hz}, 1 \mathrm{H}), 7.61(\mathrm{dt}$, $J=7.2,1.2 \mathrm{~Hz}, 1 \mathrm{H}), 7.51(\mathrm{t}, J=7.8 \mathrm{~Hz}, 1 \mathrm{H}), 7.18-7.13(\mathrm{~m}, 2 \mathrm{H}), 7.07-7.02(\mathrm{~m}, 4 \mathrm{H}), 6.81(\mathrm{~d}, J=$ $8.4 \mathrm{~Hz}, 1 \mathrm{H}), 2.33(\mathrm{~s}, 3 \mathrm{H}), 2.23(\mathrm{~s}, 3 \mathrm{H})$ - signal of the residual solvent (acetone) is visible in spectrum. ${ }^{13} \mathrm{C}\{1 \mathrm{H}\}$ NMR $\left(151 \mathrm{MHz}, \mathrm{DMSO}-d_{6}\right) \delta 168.2,153.8,149.7,140.4,135.9,131.8,131.1$, 129.2, 129.0, 128.7, 128.4, 127.4, 125.6, 125.3, 125.2, 123.2, 117.3, 68.7, 20.5, 17.0. Mp 235.2$237.2^{\circ} \mathrm{C} . v_{\max }$ (neat): $3074,2358,1680,1237,697 \mathrm{~cm}^{-1}$. HRMS (MALDI TOF) $\mathrm{m} / z:[\mathrm{M}+\mathrm{H}]^{+}$ calcd for $\mathrm{C}_{22} \mathrm{H}_{20} \mathrm{NO}_{2} \mathrm{~S} 362.1215$; found 362.1211.

3-(5-Chloro-2-hydroxyphenyl)-3-(naphthalen-2-yl)isoindolin-1-one (24). Colorless solid. Yield: $58 \mathrm{mg}(69 \%)$. Column chromatography eluents: dichloromethane-acetone 20:1. ${ }^{1} \mathrm{H}$ NMR (300 MHz, DMSO- $\left.d_{6}\right) \delta 10.07$ (s, 1H), 9.22 (s, 1H), 7.87-7.84 (m, 5H), 7.69-7.62 (m, 2H), $7.55(\mathrm{dt}, J$ $=7.2,0.9 \mathrm{~Hz}, 1 \mathrm{H}), 7.49-7.43(\mathrm{~m}, 2 \mathrm{H}), 7.33(\mathrm{dd}, J=8.7,1.8 \mathrm{~Hz}, 1 \mathrm{H}), 7.26(\mathrm{dd}, J=8.4,2.7 \mathrm{~Hz}$, $1 \mathrm{H}), 7.19(\mathrm{~d}, J=2.4 \mathrm{~Hz}, 1 \mathrm{H}), 6.87(\mathrm{~d}, J=8.7 \mathrm{~Hz}, 1 \mathrm{H}) .{ }^{13} \mathrm{C}\{1 \mathrm{H}\} \mathrm{NMR}\left(75 \mathrm{MHz}\right.$, DMSO- $\left.d_{6}\right) \delta$ $168.4,154.5,149.3,140.5,132.7,132.2,132.1,131.2,130.1,129.1,128.8,128.00,128.95,127.4$ $126.9,126.3,126.0,125.3,124.1,123.5,123.4,122.1,118.2,68.9 . M p 283.6-286.5^{\circ} \mathrm{C} . v_{\max }$ (neat): 3053, 1659, 1416, 1118, 700, $556 \mathrm{~cm}^{-1}$. HRMS (MALDI TOF) $\mathrm{m} / z:[\mathrm{M}+\mathrm{H}]^{+}$calcd for $\mathrm{C}_{24} \mathrm{H}_{17} \mathrm{ClNO}_{2} 386.0870$; found 386.0879.

5,6-Dichloro-3-(5-chloro-2-hydroxyphenyl)-3-(3-methoxyphenyl)isoindolin-1-one (25). Colorless solid. Yield: $75 \mathrm{mg}$ (79\%). Column chromatography eluents: dichloromethane-acetone 20:1. ${ }^{1} \mathrm{H}$ 
NMR (600 MHz, DMSO- $\left.d_{6}\right) \delta 10.8$ (brs, 1H), $9.54(\mathrm{~s}, 1 \mathrm{H}), 8.20(\mathrm{~s}, 1 \mathrm{H}), 7.90(\mathrm{~s}, 1 \mathrm{H}), 7.23(\mathrm{dt}, J$ $=8.1,2.1 \mathrm{~Hz}, 2 \mathrm{H}), 7.15(\mathrm{~d}, J=2.7 \mathrm{~Hz}, 1 \mathrm{H}), 6.87(\mathrm{dd}, J=8.1,2.4 \mathrm{~Hz}, 1 \mathrm{H}), 6.83(\mathrm{~d}, J=9.6 \mathrm{~Hz}$, 1H), $6.76(\mathrm{dd}, J=7.8,0.9 \mathrm{~Hz}, 1 \mathrm{H}), 6.70(\mathrm{t}, J=1.8 \mathrm{~Hz}, 1 \mathrm{H}), 3.68(\mathrm{~s}, 3 \mathrm{H}) .{ }^{13} \mathrm{C}\{1 \mathrm{H}\} \mathrm{NMR}(151$ MHz, DMSO- $\left.d_{6}\right) \delta 166.3,159.3,154.3,149.5,143.4,135.1,132.3,132.1,129.8,129.5,129.3$, 127.2, 127.1, $125.3,122.4,118.3,118.2,112.4,112.3,68.6,55.2 . M p 151.7-153.6{ }^{\circ} \mathrm{C} . v_{\max }$ (neat): 2996, 2360, 1597, 1411, 1253, 776, 682, $466 \mathrm{~cm}^{-1}$. HRMS (MALDI TOF) $\mathrm{m} / z:[\mathrm{M}+\mathrm{H}]^{+}$calcd for $\mathrm{C}_{21} \mathrm{H}_{15} \mathrm{Cl}_{3} \mathrm{NO}_{3}$ 434.0039; found 434.0029 .

3-(4-(Diethylamino)-2-hydroxyphenyl)-3-(4-fluorophenyl)isoindolin-1-one (26). Colorless solid. Yield: $56 \mathrm{mg}(66 \%)$. Column chromatography eluents: dichloromethane-acetone 50:1. ${ }^{1} \mathrm{H}$ NMR $\left(600 \mathrm{MHz}, \mathrm{DMSO}-d_{6}\right) \delta 9.32(\mathrm{~s}, 1 \mathrm{H}), 8.75(\mathrm{~s}, 1 \mathrm{H}), 7.68(\mathrm{~d}, J=7.2 \mathrm{~Hz}, 1 \mathrm{H}), 7.62(\mathrm{~d}, J=7.2 \mathrm{~Hz}$, 1H), $7.59(\mathrm{t}, J=7.2 \mathrm{~Hz}, 1 \mathrm{H}), 7.48(\mathrm{t}, J=7.2 \mathrm{~Hz}, 1 \mathrm{H}), 7.19(\mathrm{dt}, J=5.4,3.0 \mathrm{~Hz}, 2 \mathrm{H}), 7.06(\mathrm{t}, J=$ $9.0 \mathrm{~Hz}, 2 \mathrm{H}), 6.86(\mathrm{~d}, J=9.0 \mathrm{~Hz}, 1 \mathrm{H}), 6.16(\mathrm{~d}, J=1.8 \mathrm{~Hz}, 1 \mathrm{H}), 6.03(\mathrm{dd}, J=9.0,1.8 \mathrm{~Hz}, 1 \mathrm{H}), 3.23$ $(\mathrm{q}, J=7.2 \mathrm{~Hz}, 4 \mathrm{H}), 1.05(\mathrm{t}, J=6.6 \mathrm{~Hz}, 6 \mathrm{H}) .{ }^{13} \mathrm{C}\{1 \mathrm{H}\} \mathrm{NMR}\left(151 \mathrm{MHz}, \mathrm{DMSO}-d_{6}\right) \delta 168.2,161.1$ $(\mathrm{d}, J=242 \mathrm{~Hz}), 156.06,150.4,148.5,140.7,131.6,131.0,128.1,127.9,127.4(\mathrm{~d}, J=8.0 \mathrm{~Hz})$, 125.3, 123.2, 114.7, 114.6, 101.6, 99.4, 68.2, 43.6, 12.5. Mp 269.9-298.3 ${ }^{\circ} \mathrm{C} . v_{\max }$ (neat): 3422 , $3154,1685,1323,1220,1071,927,845,708 \mathrm{~cm}^{-1}$. HRMS (MALDI TOF) $\mathrm{m} / z:[\mathrm{M}+\mathrm{H}]^{+}$calcd for $\mathrm{C}_{24} \mathrm{H}_{24} \mathrm{FN}_{2} \mathrm{O}_{2} 391.1822$; found 391.1827 .

3-(4-Fluorophenyl)-3-(4-hydroxyphenyl)isoindolin-1-one (27). Colorless solid. 3-(4fluorophenyl)-3-hydroxyisoindolin-1-one 41 (150 $\mathrm{mg}, 0.66 \mathrm{mmol})$ afforded $202 \mathrm{mg}$ (96\%) of the title compound. Column chromatography eluents: dichloromethane-acetone 5:1. ${ }^{1} \mathrm{H}$ NMR (600 MHz, DMSO- $\left.d_{6}\right) \delta 9.58(\mathrm{~s}, 1 \mathrm{H}), 9.45(\mathrm{brs}, 1 \mathrm{H}), 7.70(\mathrm{~d}, J=7.2 \mathrm{~Hz}, 1 \mathrm{H}), 7.63-7.57(\mathrm{~m}, 2 \mathrm{H}), 7.51$ (t, $J=7.2 \mathrm{~Hz}, 1 \mathrm{H}), 7.30(\mathrm{~m}, 2 \mathrm{H}), 7.15(\mathrm{t}, J=9.0 \mathrm{~Hz}, 2 \mathrm{H}), 7.02(\mathrm{~d}, J=8.4 \mathrm{~Hz}, 2 \mathrm{H}), 6.72(\mathrm{~d}, J=8.4$ $\mathrm{Hz}, 2 \mathrm{H}) .{ }^{13} \mathrm{C}\{1 \mathrm{H}\} \mathrm{NMR}\left(151 \mathrm{MHz}, \mathrm{DMSO}-d_{6}\right) \delta 168.1,162.1,160.5,156.7,150.2,139.8(\mathrm{~d}, J=$ 
$3.0 \mathrm{~Hz}), 133.1,131.9,131.0,128.9(\mathrm{~d}, J=8.3 \mathrm{~Hz}), 128.3,128.0,124.5,123.1,115.0,114.8,69.2$ - signal of the residual solvent (acetone) is visible in spectra. Mp 144.0-144.9 ${ }^{\circ} \mathrm{C} . v_{\max }$ (neat): 3167, 1682, 1289, 1217, 962, $708 \mathrm{~cm}^{-1}$. HRMS (MALDI TOF) $\mathrm{m} / \mathrm{z}:[\mathrm{M}+\mathrm{H}]^{+}$calcd for $\mathrm{C}_{20} \mathrm{H}_{15} \mathrm{FNO}_{2} 320.1087$; found 320.1052 .

3-(5-Bromo-2-methoxyphenyl)-3-phenylisoindolin-1-one (29). Prepared according to a modified known procedure. ${ }^{15}$ To a solution of $\mathbf{1 1}(100 \mathrm{mg}, 0.26 \mathrm{mmol})$ in acetone $(2.0 \mathrm{~mL}), \mathrm{K}_{2} \mathrm{CO}_{3}(180$ $\mathrm{mg}, 1.3 \mathrm{mmol})$ and $\mathrm{MeI}(21 \mu \mathrm{L}, 0.34 \mathrm{mmol})$ were added and the resulting mixture was refluxed for 24 hours in an oil bath. Then, the solvent was evaporated and column chromatography of the residue on silica gel (DCM/acetone 90:1) afforded $67 \mathrm{mg}$ (65\%) of the title compound as colorless solid. ${ }^{1} \mathrm{H}$ NMR (300 MHz, DMSO- $\left.d_{6}\right) \delta 9.29(\mathrm{~s}, 1 \mathrm{H}), 7.74-7.68(\mathrm{~m}, 2 \mathrm{H}), 7.64(\mathrm{dt}, J=7.8,1.2 \mathrm{~Hz}$, 1H), 7.56-7.51 (m, 2H), 7.32-7.21 (m, 4H), 7.15-7.12 (m, 2H), $7.05(\mathrm{~d}, J=8.7 \mathrm{~Hz}, 1 \mathrm{H}), 3.47(\mathrm{~s}$, $3 \mathrm{H}) .{ }^{13} \mathrm{C}\{1 \mathrm{H}\} \operatorname{NMR}\left(75 \mathrm{MHz}, \mathrm{DMSO}-d_{6}\right) \delta 168.3,156.6,148.9,143.1,132.7,132.2,132.1,131.1$, $129.4,128.8,128.3,127.0,125.2,124.8,123.5,115.1,111.5,68.6,55.74$ - signals of inseparable impurities are visible in spectra. $\mathrm{Mp} 263.1-265.0^{\circ} \mathrm{C} . v_{\max }$ (neat): $3166,1682,1289,1217,938$, $707 \mathrm{~cm}^{-1}$. HRMS (MALDI TOF) $\mathrm{m} / \mathrm{z}$ : $[\mathrm{M}+\mathrm{H}]^{+}$calcd for $\mathrm{C}_{21} \mathrm{H}_{17} \mathrm{BrNO}_{2}$ 394.0443; found 394.0454.

4-Chloro-2-(1-phenylisoindolin-1-yl)phenol (30). $\mathrm{LiAlH}_{4}(26 \mathrm{mg}, 0.07 \mathrm{mmol})$ was added to a solution of compound $\mathbf{1}(35 \mathrm{mg}, 0.10 \mathrm{mmol})$ in freshly distilled THF $(2.0 \mathrm{~mL})$ at $0{ }^{\circ} \mathrm{C}$. After 24 hours at reflux in an oil bath, reaction mixture was quenched with saturated aqueous solution of Rochelle salt $(5 \mathrm{~mL})$ and extracted with DCM $(3 \times 20 \mathrm{~mL})$. Organic layers were collected, dried over $\mathrm{Na}_{2} \mathrm{SO} 4$, filtered and evaporated under reduced pressure. Column chromatography of the residue on silica gel (DCM/acetone 5:1) afforded $27 \mathrm{mg}(84 \%)$ of the title compound as colorless solid. ${ }^{1} \mathrm{H}$ NMR (600 MHz, DMSO- $\left.d_{6}\right) \delta$ 7.41-7.40 (m, 1H), 7.37-7.30 (m, 6H), 7.14-7.12 (m, 2H), 
7.07-7.06 (m, 3H), $4.10(\mathrm{dd}, J=19.8,14.4 \mathrm{~Hz}, 2 \mathrm{H})-\mathrm{N} H$ and $\mathrm{OH}$ protons are observable in ${ }^{1} \mathrm{H}$ NMR spectrum but have low intensity. ${ }^{13} \mathrm{C}\{1 \mathrm{H}\}$ NMR (151 MHz, DMSO- $\left.d_{6}\right) \delta 157.9,144.5$, $143.3,141.1,128.5,128.4,128.4,127.8,127.7,127.6,127.2,124.5,122.9,121.1,118.8,76.5$, 48.8 - one signal in aromatic region is overlapped. Mp 177.1-179.0 ${ }^{\circ} \mathrm{C} . v_{\max }$ (neat): 3167,1682 , 1289 1217, 938, $707 \mathrm{~cm}^{-1}$. HRMS (MALDI TOF) $\mathrm{m} / z:[\mathrm{M}+\mathrm{H}]^{+}$calcd for $\mathrm{C}_{20} \mathrm{H}_{17} \mathrm{ClNO}_{2} 322.0999$; found 322.0985 .

4-(1-(4-Fluorophenyl)isoindolin-1-yl)phenol (31). $\mathrm{LiAlH}_{4}(26 \mathrm{mg}, 0.07 \mathrm{mmol})$ was added to a solution of compound $27(32 \mathrm{mg}, 0.10 \mathrm{mmol})$ in freshly distilled THF $(2.0 \mathrm{~mL})$ at $0{ }^{\circ} \mathrm{C}$. After 24 hours at reflux in an oil bath, reaction mixture was quenched with saturated aqueous solution of Rochelle salt $(5 \mathrm{~mL})$ and extracted with DCM (3 x $20 \mathrm{~mL})$. Organic layers were collected, dried over $\mathrm{Na}_{2} \mathrm{SO} 4$, filtered and evaporated under reduced pressure. Column chromatography of the residue on silica gel (DCM/acetone 5:1) afforded $29 \mathrm{mg}(96 \%)$ of the title compound as colorless solid. ${ }^{1} \mathrm{H}$ NMR (600 MHz, DMSO-d6) $\delta 9.33(\mathrm{~s}, 1 \mathrm{H})$, 7.36-7.34 (m, 2H), 7.28-7.26 (m, 1H), 7.23$7.21(\mathrm{~m}, 3 \mathrm{H}), 7.10-7.07(\mathrm{~m}, 2 \mathrm{H}), 7.01-6.99(\mathrm{~m}, 2 \mathrm{H}), 6.68-6.65(\mathrm{~m}, 2 \mathrm{H}), 4.08(\mathrm{dd}, J=27.6,14.4$ $\mathrm{Hz}, 2 \mathrm{H})-\mathrm{N} H$ signal is not visible in ${ }^{1} \mathrm{H}$ NMR spectrum. ${ }^{13} \mathrm{C}\{1 \mathrm{H}\} \mathrm{NMR}\left(151 \mathrm{MHz}, \mathrm{DMSO}-d_{6}\right) \delta$ $160.9(\mathrm{~d}, J=243.0 \mathrm{~Hz}), 156.1,147.6,143.3(\mathrm{~d}, J=2.7 \mathrm{~Hz}), 142.3,136.9,129.4(\mathrm{~d}, J=8.0 \mathrm{~Hz})$, 128.6, 126.8, 126.6, 124.2, 122.4, 115.2, 114.6, 114.5 (d, J=20.9 Hz), 74.9, 50.2. Mp 183.1-184.2 ${ }^{\circ} \mathrm{C} . v_{\max }$ (neat): 2584, 2015, 1506, 1313, 1256, 852, 827, $603 \mathrm{~cm}^{-1}$. HRMS (MALDI TOF) m/z: [M $+\mathrm{H}]^{+}$calcd for $\mathrm{C}_{20} \mathrm{H}_{17} \mathrm{FNO} 306.1294$; found 306.1295.

\section{Associated content}

Supporting Information: NMR spectra, list of starting 3-aryl 3-hydroxyisoindolinones, 2D NMR experiments, temperature variation experiments for compound $\mathbf{6}$. 
FAIR Data is available as Supporting Information for Publication and includes the primary NMR FID files for all synthesized compounds.

\section{Author Information}

Corresponding author

Matija Gredičak - Division of Organic Chemistry and Biochemistry, Ruđer Bošković Institute, Bijenička c. 54, 10000 Zagreb, Croatia; ORCID: 0000-0002-6306-5210

Email: matija.gredicak@irb.hr

$\underline{\text { Authors }}$

Danijel Glavač - Division of Organic Chemistry and Biochemistry, Ruđer Bošković Institute, Bijenička c. 54, 10000 Zagreb, Croatia; ORCID 0000-0001-6737-0629

Nikola Topolovčan - Division of Organic Chemistry and Biochemistry, Ruđer Bošković Institute, Bijenička c. 54, 10000 Zagreb, Croatia; ORCID 0000-0003-3417-7368

\section{Notes}

The manuscript was written through contributions of all authors. All authors have given approval to the final version of the manuscript. The authors declare no competing financial interest.

† These authors contributed equally.

\section{Acknowledgements}

Financial support was provided by the Croatian Science Foundation (grant no. IP-2018-01-4053).

\section{References}


1. (a) Kovacic, P.; Somanathan, R. Clinical physiology and mechanism of dizocilpine (MK801): Electron transfer, radicals, redox metabolites and bioactivity. Oxid. Med. Cell. Longev. 2010, 3, 13-22. (b) Phenytoin https://www.drugs.com/phenytoin.html. (c) Mathew, L.; Katz, S.D. Calcium Sensitising Agents in Heart Failure. Drug Aging 1998, 12, 191-204. (d) Jacobson, A.; Scott, M. WO2009045761, 2009.

2. (a) Kim, H. N.; Lee, M. H.; Kim, H. J.; Kim, J. S.; Yoon, J. A new trend in rhodaminebased chemosensors: Application of spirolactam ring-opening to sensing ions. Chem. Soc. Rev. 2008, 37, 1465-1472. (b) Sun, J.; Li, X.; Cao, J.; Sun, Q.; Zhang, Y.; Wang, X.; Wu, T.; Hu, X.; Feng, F. Mitochondria Targeting Fluorescent Probes Based on through BondEnergy Transfer for Mutually Imaging Signaling Molecules $\mathrm{H}_{2} \mathrm{~S}$ and $\mathrm{H}_{2} \mathrm{O}_{2}$. Chem. Eur. J. 2019, 25, 9164-9169. (c) Yang, G.; Meng, X.; Fang, S.; Wang, L.; Wang, Z.; Wang, F.; Duan, H.; Hao, A. Two novel pyrazole-based chemosensors: "naked-eye" colorimetric recognition of $\mathrm{Ni}^{2+}$ and $\mathrm{Al}^{3+}$ in alcohol and aqueous DMF media. New J. Chem. 2018, 42, 14630-14641. (d) Gao, M. X.; Zou, H. Y.; Li, Y. F.; Huang, C. Z. General Sensitive Detecting Strategy of Ions through Plasmonic Resonance Energy Transfer from Gold Nanoparticles to Rhodamine Spirolactam. Anal. Chem. 2017, 89, 1808-1814. (e) Lohar, S.; Banerjee, A.; Sahana, A.; Panja, S.; Hauli, I.; Mukhopadhyay, S. K.; Das, D. Selective fluorescence and naked eye detection of histidine in aqueous medium via hydrogen bonding assisted Schiff base condensation. Tetrahedron Lett. 2014, 55, 174-176. (f) Fan, J.; Zhan, P.; Hu, M.; Sun, W.; Tang, J.; Wang, J.; Sun, S.; Song, F.; Peng, X. A fluorescent ratiometric chemodosimeter for $\mathrm{Cu}^{2+}$ based on TBET and its application in living cells. Org. Lett. 2013, 15, 492-495.

3. (a) Qi, Q.; Chi, W.; Li, Y.; Qiao, Q.; Chen, J.; Miao, L.; Zhang, Y.; Li, J.; Ji, W.; Xu, T.; Liu, X.; Yoon, J.; Xu, Z. A H-bond strategy to develop acid-resistant photoswitchable 
rhodamine spirolactams for super-resolution single-molecule localization microscopy. Chem. Sci. 2019, 10, 4914-4922. (b) Ye, Z.; Yu, H.; Yang, W.; Zheng, Y.; Li, N.; Bian, H.; Wang, Z.; Liu, Q.; Song, Y.; Zhang, M.; Xiao, Y. Strategy to Lengthen the On-Time of Photochromic Rhodamine Spirolactam for Super-resolution Photoactivated Localization Microscopy. J. Am. Chem. Soc. 2019, 141, 6527-6536. (c) Roubinet, B.; Bischoff, M.; Nizamov, S.; Yan, S.; Geisler, C.; Stoldt, S.; Mitronova, G. Y.; Belov, V. N.; Bossi, M. L.; Hell, S. W. Photoactivatable Rhodamine Spiroamides and Diazoketones Decorated with "universal Hydrophilizer" or Hydroxyl Groups. J. Org. Chem. 2018, 83, 6466-6476. (d) Lee, M. K.; Rai, P.; Williams, J.; Twieg, R. J.; Moerner, W. E. Small-molecule labeling of live cell surfaces for three-dimensional super-resolution microscopy. J. Am. Chem. Soc. 2014, 136, 14003-14006. (e) Fölling, J.; Belov, V.; Kunetsky, R.; Medda, R.; Schönle, A.; Egner, A.; Eggeling, C.; Bossi, M.; Hell, S. W. Photochromic rhodamines provide nanoscopy with optical sectioning. Angew. Chem. Int. Ed. 2007, 46, 6266-6270. (f) Belov, V. N.; Bossi, M. L.; Fölling, J.; Boyarskiy, V. P.; Hell, S. W. Rhodamine Spiroamides for Multicolor Single-Molecule Switching Fluorescent Nanoscopy. Chem. Eur. J. 2009, 15, $10762-10776$.

4. (a) Wu, Y.; Zhang, Y.; Jiang, M.; Dong, X.; Jalani, H. B.; Li, G.; Lu, H. Synergistic combination of visible-light photo-catalytic electron and energy transfer facilitating multicomponent synthesis of $\beta$-functionalized $\alpha, \alpha$-diarylethylamines. Chem. Commun. 2019, 55, 6405-6408. (b) Desmarchelier, A.; Ortiz, P.; Harutyunyan, S. R. Tertiary $\alpha-$ diarylmethylamines derived from diarylketimines and organomagnesium reagents. Chem. Commun. 2015, 51, 703-706. (c) Jackl, M. K.; Schuhmacher, A.; Shiro, T.; Bode, J. W. Synthesis of $N, N$-Alkylated $\alpha$-Tertiary Amines by Coupling of $\alpha$ Aminoalkyltrifluoroborates and Grignard Reagents. Org. Lett. 2018, 20, 4044-4047. (d) 
Tait, M.; Donnard, M.; Minassi, A.; Lefranc, J.; Bechi, B.; Carbone, G.; O’Brien, P.; Clayden, J. Amines bearing tertiary substituents by tandem enantioselective carbolithiation-rearrangement of vinylureas. Org. Lett. 2013, 15, 34-37. (e) Kong, J.; McLaughlin, M.; Belyk, K.; Mondschein, R. Enantioselective Rh(I)-Catalyzed Addition of Arylboronic Acids to Cyclic Ketimines. Org. Lett. 2015, 17, 5520-5523.

5. (a) Cui, Y.; Yamashita, Y.; Kobayashi, S. Facile preparation of allylzinc species from allylboronates and zinc amide via a boron-to-zinc exchange process and their reactions with carbonyl compounds, imines and hydrazones. Chem. Commun. 2012, 48, 1031910321. (b) Cui, Y.; Li, W.; Sato, T.; Yamashita, Y.; Kobayashi, S. Catalytic use of zinc amide for transmetalation with allylboronates: General and efficient catalytic allylation of carbonyl compounds, imines, and hydrazones. Adv. Synth. Catal. 2013, 355, 1193-1205. (c) Glavač, D.; Zheng, C.; Dokli, I.; You, S.-L.; Gredičak, M. Chiral Brønsted Acid Catalyzed Enantioselective aza-Friedel-Crafts Reaction of Cyclic $\alpha$-Diaryl $N$-Acyl Imines with Indoles. J. Org. Chem. 2017, 82, 8752-8760.

6. Li, M.; Berritt, S.; Matuszewski, L.; Deng, G.; Pascual-Escudero, A.; Panetti, G. B.; Poznik, M.; Yang, X.; Chruma, J. J.; Walsh, P. J. Transition-Metal-Free Radical C(sp $\left.{ }^{3}\right)-$ $\mathrm{C}\left(\mathrm{sp}^{2}\right)$ and $\mathrm{C}\left(\mathrm{sp}^{3}\right)-\mathrm{C}\left(\mathrm{sp}^{3}\right)$ Coupling Enabled by 2-Azaallyls as Super-Electron-Donors and Coupling-Partners. J. Am. Chem. Soc. 2017, 139, 16327-16333.

7. (a) Nishimura, T.; Noishiki, A.; Chit Tsui, G.; Hayashi, T. Asymmetric synthesis of (Triaryl)methylamines by rhodium-catalyzed addition of arylboroxines to cyclic $\mathrm{N}$ sulfonyl ketimines. J. Am. Chem. Soc. 2012, 134, 5056-5059. (b) Wang, H.; Jiang, T.; Xu, M.-H. Simple Branched Sulfur-Olefins as Chiral Ligands for Rh-Catalyzed Asymmetric Arylation of Cyclic Ketimines: Highly Enantioselective Construction of Tetrasubstituted Carbon Stereocenters. J. Am. Chem. Soc. 2013, 135, 971-974. 
8. (a) Yang, G.; Zhang, W. A. A palladium-catalyzed enantioselective addition of arylboronic acids to cyclic ketimines. Angew. Chem. Int. Ed. 2013, 52, 7540-7544. (b) Jiang, C.; Lu, Y.; Hayashi, T. High performance of a palladium phosphinooxazoline catalyst in the asymmetric arylation of cyclic $N$-sulfonyl ketimines. Angew. Chem. Int. Ed. 2014, 53, 9936-9939. (c) Álvarez-Casao, Y.; Monge, D.; Álvarez, E.; Fernández, R.; Lassaletta, J. M. Pyridine-hydrazones as $N, N^{\prime}$-Ligands in Asymmetric Catalysis: $\operatorname{Pd}(\mathrm{II})$-Catalyzed Addition of Boronic Acids to Cyclic Sulfonylketimines. Org. Lett. 2015, 17, 5104-5107. (c) Zhou, B.; Li, K.; Jiang, C.; Lu, Y.; Hayashi, T. Modified Amino Acid-Derived Phosphine-Imine Ligands for Palladium-Catalyzed Asymmetric Arylation of Cyclic $\mathrm{N}$ Sulfonyl Imines. Adv. Synth. Catal. 2017, 359, 1969-1975.

9. Nishimura, T.; Noishiki, A.; Ebe, Y.; Hayashi, T. Hydroxorhodium/Chiral Diene Complexes as Effective Catalysts for the Asymmetric Arylation of 3-Aryl-3hydroxyisoindolin-1-ones. Angew. Chem. Int. Ed. 2013, 52, 1777-1780.

10. (a) Olyaei, A.; Sadeghpour, M. Recent advances in the synthesis and synthetic applications of Betti base (aminoalkylnaphthol) and bis-Betti base derivatives. RSC Adv. 2019, 9, 18467-18497. and references cited therein. (b) Cardellicchio, C.; Capozzi, M. A. M.; Naso, F. The Betti base: the awakening of a sleeping beauty. Tetrahedron Asymmetry 2010, $21,507-517$.

11. (a) Gao, H.; Sun, J.; Yan, C. G. Synthesis of new type of Betti bases via three-component reaction of $\beta$-naphthol, cyclic amines and isatins. Chin. Chem. Lett. 2015, 26, 353-356. (b) Montesinos-Magraner, M.; Vila, C.; Cantõn, R.; Blay, G.; Fernández, I.; Muñoz, M. C.; Pedro, J. R. Organocatalytic asymmetric addition of naphthols and electron-rich phenols to isatin-derived ketimines: Highly enantioselective construction of tetrasubstituted stereocenters. Angew. Chem. Int. Ed. 2015, 54, 6320-6324. (c) Kumari, P.; Barik, S.; Khan, 
N. H.; Ganguly, B.; Kureshy, R. I.; Abdi, S. H. R.; Bajaj, H. C. The origin for highly enantioselective induction of 1-naphthol to isatin-derived N-Boc ketimines catalyzed by quinine thiourea catalyst: An experimental and computational study. $R S C A d v$. 2015, 5, 69493-69501. (c) Zhou, D.; Huang, Z.; Yu, X.; Wang, Y.; Li, J.; Wang, W.; Xie, H. A Quinine-Squaramide Catalyzed Enantioselective Aza-Friedel-Crafts Reaction of Cyclic Trifluoromethyl Ketimines with Naphthols and Electron-Rich Phenols. Org. Lett. 2015, $17,5554-5557$.

12. (a) Basson, A. J.; McLaughlin, M. G. Synthesis of Functionalized Isoindolinones via Calcium Catalyzed Generation and Trapping of N-Acyliminium Ions. J. Org. Chem. 2020, 85, 5615-5628. (b) Das, D. K.; Kannaujiya, V. K.; Sadhu, M. M.; Ray, S. K.; Singh, V. K. $\mathrm{BF}_{3} \cdot \mathrm{OEt}_{2}$-Catalyzed Vinyl Azide Addition to in Situ Generated N-Acyl Iminium Salts: Synthesis of 3-Oxoisoindoline-1-acetamides. J. Org. Chem. 2019, 84, 15865-15876. (c) Unhale, R. A.; Sadhu, M. M.; Ray, S. K.; Biswas, R. G.; Singh, V. K. A chiral Brønsted acid-catalyzed highly enantioselective Mannich-type reaction of $\alpha$-diazo esters with in situ generated $N$-acyl ketimines. Chem. Commun. 2018, 54, 3516-3519. (d) Kang, Z.; Zhang, D.; Shou, J.; Hu, W. Enantioselective Trapping of Oxonium Ylides by 3Hydroxyisoindolinones via a Formal SN1 Pathway for Construction of Contiguous Quaternary Stereocenters. Org. Lett. 2018, 20, 983-986. (e) Zhang, L.; Wu, B.; Chen, Z.; Hu, J.; Zeng, X.; Zhong, G. Chiral phosphoric acid catalyzed enantioselective $N$-alkylation of indoles with in situ generated cyclic $N$-acyl ketimines. Chem. Commun. 2018, 54, 92309233. (f) Suć, J.; Dokli, I.; Gredičak, M. Chiral Brønsted acid-catalysed enantioselective synthesis of isoindolinone-derived $N($ acyl $), S$-acetals. Chem. Commun. 2016, 52, 20712074. (g) Unhale, R. A.; Molleti, N.; Rana, N. K.; Dhanasekaran, S.; Bhandary, S.; Singh, V. K. Chiral phosphoric acid catalyzed enantioselective addition of thiols to in situ 
generated ketimines: Synthesis of N,S-ketals. Tetrahedron Lett. 2017, 58, 145-151. (h) Suneja, A.; Unhale, R. A.; Singh, V. K. Enantioselective Hydrophosphonylation of in Situ Generated N-Acyl Ketimines Catalyzed by BINOL-Derived Phosphoric Acid. Org. Lett. 2017, 19, 476-479. (i) Glavač, D.; Jerić, I.; Gredičak, M. Organocatalytic synthesis of $\alpha, \alpha-$ diaryl substituted $\alpha$-amino acid derivatives by an interrupted three-component Ugi reaction. Croat. Chem. Acta 2019, 92, 203-209. (j) Chen, M.-W.; Chen, Q.-A.; Duan, Y.; Ye, Z.-S.; Zhou, Y.-G. Asymmetric hydrogenolysis of racemic tertiary alcohols, 3substituted 3-hydroxyisoindolin-1-ones. Chem. Commun. 2012, 48, 1698-1700.

13. Kaboudin, B.; Abedi, Y. A novel synthesis of aryl mesylates via one-pot demethylationmesylation of aryl methyl ethers using a mixture of phosphorus pentoxide in methanesulfonic acid. Synthesis 2009, No. 12, 2025-2028.

14. Glavač, D.; Dokli, I.; Gredičak, M. Synthesis of 3-aryl 3-hydroxyisoindolinones by the Addition of Grignard and Organolithium Reagents to Phthalimides. Curr. Org. Chem. 2017, 21, 1335-1340.

15. An, P.; Shi, Z-F.; Dou, W.; Cao, X-P.; Zhang, H-L. Synthesis of 1,4-Bis[2,2-bis(4alkoxyphenyl)vinyl]benzenes and Side Chain Modulation of Their Solid-State Emission Org. Lett. 2010, 12, 4364-4367. 